\title{
Chronic Spontaneous Activity Generated in the Somata of Primary Nociceptors Is Associated with Pain-Related Behavior after Spinal Cord Injury
}

\author{
Supinder S. Bedi, ${ }^{1 \star}$ Qing Yang, ${ }^{1 *}$ Robyn J. Crook, ${ }^{1 \star}$ Junhui Du, ${ }^{2}$ Zizhen Wu, ${ }^{1}$ Harvey M. Fishman, ${ }^{1}$ Raymond J. Grill, ${ }^{1}$ \\ Susan M. Carlton, ${ }^{2}$ and Edgar T. Walters ${ }^{1}$ \\ ${ }^{1}$ Department of Integrative Biology and Pharmacology, University of Texas Medical School at Houston, Houston, Texas 77030, and ${ }^{2}$ Department of \\ Neuroscience and Cell Biology, University of Texas Medical Branch, Galveston, Texas 77555
}

\begin{abstract}
Mechanisms underlying chronic pain that develops after spinal cord injury (SCI) are incompletely understood. Most research on SCI pain mechanisms has focused on neuronal alterations within pain pathways at spinal and supraspinal levels associated with inflammation and glial activation. These events might also impact central processes of primary sensory neurons, triggering in nociceptors a hyperexcitable state and spontaneous activity (SA) that drive behavioral hypersensitivity and pain. SCI can sensitize peripheral fibers of nociceptors and promote peripheral SA, but whether these effects are driven by extrinsic alterations in surrounding tissue or are intrinsic to the nociceptor, and whether similar SA occurs in nociceptors in vivo are unknown. We show that small DRG neurons from rats (Rattus norvegicus) receiving thoracic spinal injury $3 \mathrm{~d}$ to 8 months earlier and recorded $1 \mathrm{~d}$ after dissociation exhibit an elevated incidence of SA coupled with soma hyperexcitability compared with untreated and sham-treated groups. SA incidence was greatest in lumbar DRG neurons (57\%) and least in cervical neurons (28\%), and failed to decline over 8 months. Many sampled SA neurons were capsaicin sensitive and/or bound the nociceptive marker, isolectin B4. This intrinsic SA state was correlated with increased behavioral responsiveness to mechanical and thermal stimulation of sites below and above the injury level. Recordings from C- and A $\delta$-fibers revealed SCI-induced SA generated in or near the somata of the neurons in vivo. SCI promotes the entry of primary nociceptors into a chronic hyperexcitable-SA state that may provide a useful therapeutic target in some forms of persistent pain.
\end{abstract}

\section{Introduction}

Lifelong pain that is often debilitating and therapeutically intractable develops in many patients after spinal cord injury (SCI) (Siddall, 2009). Numerous potential mechanisms have been associated with SCI-induced pain (Finnerup and Jensen, 2004), but the primary causes of chronic pain after SCI have yet to be defined. Research has focused primarily on SCI-induced increases in responsiveness and electrical activity in central neurons within pain pathways at spinal and supraspinal levels (Waxman and Hains, 2006; Hulsebosch et al., 2009; Yezierski, 2009). These alterations are often assumed to be caused directly by a spinal cord lesion (e.g., excitation of spinal projection neurons or killing of inhibitory neurons) and indirectly by pathological central effects (e.g., deafferentation hyperexcitability of higher order neurons or glial activation).

Received May 11, 2010; revised Aug. 24, 2010; accepted Sept. 10, 2010.

This work was supported by a joint grant from The Christopher and Dana Reeve Foundation (CDRF) and the Sam Schmidt Paralysis Foundation, and National Institutes of Health (NIH) Grants NS061200 and NS35979 (E.T.W.); a postdoctoral fellowship from CDRF (S.S.B.); NIH Grants NS027910 and NS054765 and a grant from The Moody Foundation (S.M.C.); and NIH Grant NSO49409 (R.J.G.). We thank L. Lichtenberger and S. Tian for generous contributions and $\mathrm{H}$. Hu for useful comments.

*S.S.B., Q.Y., and R.J.C. contributed equally to this work.

Correspondence should be addressed to Dr. Edgar T. Walters, Department of Integrative Biology and Pharmacology, University of Texas Medical School at Houston, Houston, TX 77030. E-mail: edgar.t.walters@uth.tmc.edu. DOI:10.1523/JNEUROSCI.2428-10.2010

Copyright $\odot 2010$ the authors $\quad 0270-6474 / 10 / 3014870-13 \$ 15.00 / 0$
Another possibility is that SCI triggers persistent alterations in nociceptors like those that drive prolonged hypersensitivity and pain after serious injury and inflammation in the periphery. One potential mechanism is chronic spontaneous activity (SA) generated by hyperexcitable nociceptors. SA in primary afferents has been described in models of peripheral injury and inflammation, but the extent to which SA is intrinsic to the sensory neuron or results from continuing stimulation by extrinsic sources such as inflammatory signals is generally unknown. SA in primary afferents occurs in peripheral neuropathy (Burchiel et al., 1985; Kajander and Bennett, 1992; Amir and Devor, 1993; Study and Kral, 1996; Ali et al., 1999; Zhang et al., 1999; Djouhri et al., 2001), tissue inflammation (Koltzenburg et al., 1999; Djouhri et al., 2001; Du et al., 2003; Dang et al., 2005; Xiao and Bennett, 2007), and deep incisions (Xu and Brennan, 2010). Peripherally generated SA in primary afferents can induce and maintain central sensitization and pain (Gracely et al., 1992; Zhang et al., 2000; Sukhotinsky et al., 2004; Xie et al., 2005; Pitcher and Henry, 2008), and much of the required SA occurs in neuronal populations (C-fibers and $\mathrm{A} \delta$ fibers) containing numerous nociceptors (Wu et al., 2001; Bove et al., 2003; Djouhri et al., 2006; Xiao and Bennett, 2007; Xie et al., 2009; Xu and Brennan, 2010). DRG neurons with small somata and slowly conducting axons are especially likely to be nociceptors (Lynn and Carpenter, 1982; Koerber et al., 1988; Gold et al., 1996; Lawson, 2002; Fang et al., 2005). 
Previous SCI enhances background activity in peripheral fibers of nociceptors in an isolated skin-nerve preparation (Carlton et al., 2009), suggesting that SCI-induced SA can be generated in or near the peripheral terminals of nociceptors. However, the activity observed in this study might also have been evoked (for long periods) by the noxious stimuli used to identify each tested unit. Moreover, it is not known whether such SA is intrinsic to the nociceptor rather than driven by extrinsic signals (e.g., from altered keratinocytes) (Radtke et al., 2010), nor whether nociceptor SA occurs in vivo after SCI. Here, we show that SCI-induced SA is intrinsic to probable nociceptors, that SCI-induced SA is generated in or near the somata of these sensory neurons in vivo and in vitro, and that intrinsic SA expressed in vitro is correlated with behavioral hypersensitivity in the animals providing dissociated neurons. These results suggest that a persistent hyperexcitable-SA state in nociceptors is important not only for peripherally generated chronic pain but also for at least one form of central neuropathic pain.

\section{Materials and Methods}

All procedures complied with guidelines of the International Association for the Study of Pain (Zimmermann, 1983) and the Society for Neuroscience, and were approved by the corresponding institutional animal care and use committees at the University of Texas Medical School at Houston and the University of Texas Medical Branch in Galveston. Adult Sprague Dawley rats (200-500 g) were used, 68 males and 25 females.

\section{Behavioral tests}

Three groups of animals were tested: untreated controls (naive group), animals given sham surgery without spinal contusion (sham group), and animals receiving spinal cord injury (SCI group). Animals were maintained on a $12 \mathrm{~h}$ reverse light/dark cycle, and all behavioral and physiological tests were performed in the active (dark) phase. Animals in the SCI and sham groups were assessed for recovery of hindlimb motor function before injury, and then 1,2,3 d after injury, and finally 3-7 d before dissociating their DRG neurons 1 or 3-8 months after injury. Animals in all groups received a standard $5 \mathrm{~d}$ sequence of tests for mechanical and thermal sensitivity before injury treatment and then before the dissociation procedure. Not all animals received behavioral tests. Animals whose DRGs were harvested $3 \mathrm{~d}$ after injury were not tested behaviorally because acute aftereffects of spinal injury (spinal shock) confound behavioral responses to standard test stimuli.

Recovery of hindlimb motor function. Animals in the SCI and sham groups were assessed for hindlimb motor function while moving freely in a circular enclosure (1 m diameter). The Basso, Beattie, Bresnahan (BBB) open-field scale (Basso et al., 1996) was used, which applies detailed criteria to rate abnormalities of movement and posture, with scores ranging from 0 (no hindlimb movement) to 21 (normal hindlimb function).

Habituation to test conditions. On day 1 of the $5 \mathrm{~d}$ test sequence, animals were placed inside the Plexiglas compartments used later to test thermal and mechanical sensitivity of the paws and to test mechanical sensitivity of the torso, but no test stimuli were delivered. On day 2, the animals received tests for thermal sensitivity of the paws (after $20 \mathrm{~min}$ acclimation), but these tests were used for habituation and were not included in the analysis. On day 3 , the animals received tests (after $20 \mathrm{~min}$ acclimation) for mechanical sensitivity of the paws and torso, but these habituating tests also were omitted from the analysis. On day 4 , data were collected during tests (identical with those on day 2) of thermal sensitivity of the paws. On day 5, data were collected during tests of mechanical sensitivity of the paws and torso identical with those on day 3 .

Thermal sensitivity of paws. Thermal hypersensitivity (often used as a measure of hyperalgesia) after injury was defined as a decrease in paw withdrawal latency during a radiant heat stimulus delivered to the plantar surface of the paw (Hargreaves et al., 1988; Song et al., 2006; Carlton et al., 2009). Animals were placed in compartments on a glass floor kept at $22^{\circ} \mathrm{C}$, and a heat source (Plantar Analgesia Meter; IITC) was focused on the plantar surface of each paw. Although the postures of SCI rats sometimes differed from those of naive and sham rats, test stimuli were only delivered when the paw was flat and the plantar surface flush against the glass. No differences between the SCI animals and the naive and sham animals were evident in the area of contact with the glass during the tests given 1 month or later after injury. The thermal stimulus ended when the paw moved or after $20 \mathrm{~s}$ (to prevent possible tissue damage). All four paws were stimulated in a fixed order (left rear, right rear, left front, right front) at 5 min intervals. This sequence was conducted three times, at 20 min intervals.

Mechanical sensitivity of paws. Mechanical hypersensitivity (often used as a measure of allodynia) after injury was defined as a decrease in the threshold of paw withdrawal to application of a series of calibrated von Frey filaments (Stoelting) of different stiffness, using the "up-down" method (Dixon, 1991; Chaplan et al., 1994). All four paws were tested in the same order as the thermal tests, but only one test series was delivered to each paw.

Mechanical sensitivity of torso. Mechanical hypersensitivity of the torso after injury was defined as an increase in the incidence of behavioral responses to application of a series of mechanical stimuli to sites along the animal's torso, using methods similar to those monitoring changes after SCI in "alertness," "body withdrawal" (Cruz-Orengo et al., 2006), and vocalization (Crown et al., 2006) during stimulation of the torso. A continuous series of mechanical stimuli was delivered to a $4 \times 6$ grid on the back at $\sim 5 \mathrm{~s}$ intervals, beginning with a $20 \mathrm{mN}$ von Frey hair, followed immediately by a stiff plastic rod (Q-tip handle; $2 \mathrm{~mm}$ diameter), and finally a $250 \mathrm{mN}$ von Frey hair. Each stimulus was delivered in a set sequence, moving from the lumbar region to the cervical region and from left to right at each level (left flank, left side of back, right side of back, right flank). Depending on the animal's size and position, five or seven rather than six levels were sometimes tested. One or two tested levels were above the injury level (T10), two were close to the injury level ("atlevel"), and two to three were below the injury level. During this sequence of 60-84 stimuli, each occurrence of vocalization, moving away from the stimulus, flinching of the torso, and orientation to the stimulus was noted. Mechanical sensitivity for each animal was expressed as the percentage of all stimuli evoking vocalizations (see Fig. 5) and as the percentage of all stimuli eliciting any of the selected responses.

\section{SCI procedures}

Surgery was performed under anesthetic consisting of ketamine $(80 \mathrm{mg} /$ $\mathrm{kg})$, xylazine $(10 \mathrm{mg} / \mathrm{kg})$, and acepromazine $(0.75 \mathrm{mg} / \mathrm{kg})$ delivered intraperitoneally at a dose of $0.1 \mathrm{ml} / 100 \mathrm{~g}$. Animals receiving a spinal contusion (SCI group) first underwent a laminectomy at thoracic level 10 (T10) and the vertebral column was clamped with Adson forceps at T9 and T11. Moderate spinal contusion was produced at T10 with an Infinite Horizon injury device (Precision Systems and Instrumentation), using $150 \mathrm{kdyn}$ of force and a $1 \mathrm{~s}$ dwell time. Overlying muscles were then sutured, and the skin was closed with wound clips. Animals given sham surgery received the laminectomy and identical treatment except for the impact from the injury device. SCI animals received postoperative bladder care until neurogenic bladder function returned (usually by day 14 after injury). For $5 \mathrm{~d}$ after injury, the SCI and sham-treated animals received twice daily injections of $0.9 \%$ saline (s.c.) to maintain hydration as well as buprenorphine ( $0.02 \mathrm{mg} / \mathrm{kg}$, s.c.). Antibiotics (Baytril; $2.5 \mathrm{mg} /$ $\mathrm{kg}$ ) were injected intraperitoneally twice daily for $10 \mathrm{~d}$.

\section{Examination of dissociated DRG neurons}

Dissociation and culture of DRG neurons. Under deep anesthesia (Beuthanasia; $75 \mathrm{mg} / \mathrm{kg}$, i.p.), animals were transcardially perfused with ice-cold saline, the vertebral column was removed, and selected DRGs (L5, L4, T12, T11, T9, T8, C7, C6) were transferred to Petri dishes in ice-cold DMEM where the sheath was removed and rootlets were cut off, and each was minced with fine scissors. The ganglia were incubated in a flask in a water bath shaker for $40 \mathrm{~min}$ at $34^{\circ} \mathrm{C}$ with trypsin $(0.4 \mathrm{mg} / \mathrm{ml})$ and collagenase $\mathrm{D}(0.6 \mathrm{mg} / \mathrm{ml})$. After being centrifuged and resuspended in DMEM, the DRG fragments were triturated $\sim 20$ times through firepolished glass pipettes to dissociate individual neurons. The neurons were resuspended in $0.5 \mathrm{ml}$ of DMEM and plated at low density onto dishes coated with poly-L-lysine $(50 \mu \mathrm{g} / \mathrm{ml})$. Dishes were kept in DMEM in an incubator under $5 \% \mathrm{CO}_{2}, 95 \%$ humidity, $37^{\circ} \mathrm{C}$, before electrophysiological recording the next day. 
Recording from dissociated DRG neurons. Whole-cell patch recordings were made from small neurons (soma diameter, $\leq 30 \mu \mathrm{m}$; membrane input capacitance, $C_{\mathrm{m}}, \leq 45 \mathrm{pF}$ ) using a MultiClamp $700 \mathrm{~B}$ amplifier (Molecular Devices). Patch electrodes with a resistance of 2-5 $\mathrm{M} \Omega$ were pulled from borosilicate micropipettes (Sutter Instrument) and filled with solution containing the following (in $\mathrm{mM}$ ): $134 \mathrm{KCl}, 1.6 \mathrm{MgCl}_{2}, 13.2$ $\mathrm{NaCl}, 3$ EGTA, 9 HEPES, $4 \mathrm{Mg}$-ATP, and 0.3 Na-GTP, pH 7.2 adjusted with $\mathrm{KOH}, 300 \mathrm{mOsM}$. Electrode seal resistance on the cell was $1-10 \mathrm{G} \Omega$. Recordings were conducted during constant superfusion $(2 \mathrm{ml} / \mathrm{min})$ by extracellular solution containing the following (in mM): $140 \mathrm{NaCl}, 3 \mathrm{KCl}$, $1.8 \mathrm{CaCl}_{2}, 2 \mathrm{MgCl}_{2}, 10$ HEPES, 10 glucose, pH 7.4 adjusted with $\mathrm{NaOH}$, $320 \mathrm{mOsM}$. Signals were filtered at $1 \mathrm{kHz}$ and digitized at $10 \mathrm{kHz}$ (Digidata $1440 \mathrm{~A}$; Molecular Devices). Recordings were made at $22^{\circ} \mathrm{C}$ rather than body temperature to permit direct comparisons to subsequent studies of underlying biophysical mechanisms; the slower kinetics of conductances at this temperature facilitates the experimental separation of ionic currents. Little is known about the temperature dependence of SA in nociceptive DRG neurons, but these neurons exhibit complex temperaturedependent effects on other excitability properties (Greffrath et al., 2009). While still under voltage clamp, the Clampex Membrane Test program (Molecular Devices) was used to determine $C_{\mathrm{m}}$ and membrane resistance, $R_{\mathrm{m}}$, during a $10 \mathrm{~ms}, 5 \mathrm{mV}$ depolarizing pulse from a holding potential of $-60 \mathrm{mV}$. These values were calculated from fits to the current transients and the value of the steady-state current response. The configuration was then switched to current clamp $(0 \mathrm{pA})$ for determining other electrophysiological properties. Two to 3 min later, resting membrane potential (RMP) was measured. The minimum acceptable RMP was $-40 \mathrm{mV}$. SA was then recorded over two $30 \mathrm{~s}$ periods separated by $60 \mathrm{~s}$ without recording. Action potential (AP) properties, including thresholds, were then determined with ascending series of $2 \mathrm{~ms}$ depolarizing pulses while the neuron was held at RMP. The same tests were repeated with the neuron held at -80 and $-50 \mathrm{mV}$. While held at -50 $\mathrm{mV}$, a series of $400 \mathrm{~ms}$ pulses was delivered to determine rheobase and repetitive firing properties. Repetitive firing (number of spikes during the $400 \mathrm{~ms}$ pulse) was measured at twice the rheobase current.

\section{Markers of nociceptors in vitro}

Two markers of nociceptors were examined in a sample of small DRG neurons dissociated from SCI animals. Capsaicin receptors (TRPV1) are found in many small DRG neurons and are important for thermal and chemical nociceptive responses (Caterina et al., 2000). Capsaicin sensitivity was tested under voltage clamp by delivering a gravity-fed stream of $3 \mu \mathrm{M}$ capsaicin from a $200-\mu \mathrm{m}$-wide silica tube positioned $\sim 100 \mu \mathrm{m}$ from the soma of the neuron. Immediately before application, the capsaicin was diluted 1000-fold from a stock solution (3 mM in DMSO) using the same extracellular solution used to continuously perfuse the neurons. Live cell binding by the plant lectin, Griffonia simplicifolia isolectin B4 (IB4), a marker of "nonpeptidergic" nociceptors (Stucky and Lewin, 1999), was tested immediately before patching the neuron. IB4Alexa 594 ( $3 \mu \mathrm{g} / \mathrm{ml}$; Invitrogen) in the extracellular solution was applied for $5 \mathrm{~min}$ and washed out for at least $3 \mathrm{~min}$. A neuron was judged to be IB4-positive $\left(\mathrm{IB}^{+}{ }^{+}\right)$if it exhibited a continuous red ring around its entire perimeter when viewed at a magnification of $20 \times$.

\section{In vivo recording of $D R G$ neurons}

In vivo preparation. In vivo recordings were made from dorsal root (DR) filaments in male Sprague Dawley rats (200-250 g). Animals were anesthetized with sodium pentobarbital $(50 \mathrm{mg} / \mathrm{kg}$, i.p.), and a polyethylene tracheotomy tube $(16 \mathrm{~mm})$ was inserted through a small incision in the midtrachea and secured. A midline incision was made from T12 to S1, and the muscle and connective tissue overlying the vertebrae removed. A laminectomy from L3 to L6 exposed the spinal cord, and the dura was gently peeled back. A single bolus of $0.7 \mathrm{ml}$ of pancuronium bromide (1 $\mathrm{mg} / \mathrm{ml}$ ) was then given through a cannula (PE-60 tubing) in the external jugular vein connected to an infusion pump. Anesthesia was maintained by infusing $(3 \mathrm{ml} / \mathrm{h}$ ) a mixture of $1 \mathrm{ml}$ of pentobarbital sodium (50 $\mathrm{mg}$ ) plus $2 \mathrm{ml}$ of pancuronium bromide plus $1.7 \mathrm{ml}$ of saline. Adequacy of anesthesia was confirmed by absence of corneal and pupillary reflexes and stability of end-tidal $\mathrm{CO}_{2}$ level. The total amounts of anesthetic delivered to the SCI and sham animals were approximately equal. The tracheotomy tube was connected to a ventilator system (Harvard model 683 ) providing a mixture of room air and oxygen $(3.0 \mathrm{ml}$, 54 breaths/ min). Expired $\mathrm{CO}_{2}$ was monitored (Criticare Systems) and maintained between 2.2 and $4.5 \%$. Rectal temperature was maintained at $37^{\circ} \mathrm{C}$ by a servo-controlled heating pad under the animal. The head was stabilized in a stereotaxic frame. A warm mineral oil pool, contained by skin flaps, covered the exposed spinal cord. The temperature of the pool was $37^{\circ} \mathrm{C}$, as monitored by a thermistor.

Dorsal root recordings. Both the left and the right DRs at L4, L5, or, in a few cases, L6, were used to record SA from axons of DRG neurons. An initial cut, $\sim 1.5 \mathrm{~cm}$ central to the DRG (cut 1) (see Fig. $6 A$ ), disconnected the DR from the CNS and permitted the teasing apart of filaments from the distal side of the cut. A filament was placed on a gold wire electrode, and axonal activity was recorded extracellularly using a DAM80 differential amplifier (World Precision Instruments). Action potentials corresponding to single units in the filament were identified by template matching using a CED 1401 interface and analyzed off-line using Spike 2 (version 5.08) software. Recording began at least $30 \mathrm{~min}$ after cut 1 . To answer our major question-whether SCI increases the incidence of nociresponsive units with SA that is generated in or near the DRG in vivo - the experimental design incorporated several features that limit other types of information potentially available from this preparation. A paramount concern was to minimize stimulation of the receptive fields of the units because mechanical search stimuli can evoke ongoing, low-frequency activity in nociceptors (Carlton et al., 2009; Bove and Dilley, 2010). Thus, after cut 1 , a moderately noxious mechanical stimulus (von Frey filament, $\sim 250 \mathrm{mN}$ bending force) was applied to the ipsilateral hindlimb to see whether any nociresponsive fibers in the DR filament had receptive fields in the skin. This stimulus was applied several times to different sites until a response was observed, and then the stimulus was not delivered again for at least $8 \mathrm{~min}$, and no other types of stimuli were given. Omission of further stimulation precluded additional characterization of response properties of these units and identification of different subclasses of nociceptor. To minimize the number of peripheral stimuli delivered, we only examined units that exhibited SA during this initial recording period (i.e., after cut 1 and before cut 2). Because we did not determine the number of nociceptive units that did not display SA (which would have required extensive searching with the von Frey filament), we could not estimate the fraction out of the total number of units innervating the hindlimb that did display SA. Instead, we measured the fraction of units in each group that retained SA after subsequent disconnection from the periphery (i.e., SA generated in or near the DRG). SA during this initial phase was defined as ongoing activity occurring at least $3 \mathrm{~min}$ after the last mechanical search stimulus at a firing frequency $>0.05 \mathrm{~Hz}$, and persisting $>5 \mathrm{~min}$. To confirm that a unit displaying SA was activated by the $250 \mathrm{mN}$ von Frey hair, the corresponding receptive field was then stimulated once more before cut 2 . This cut was made to the spinal nerve $5-10 \mathrm{~mm}$ distal to the L4 or L5 DRG, eliminating peripheral input to the DRG. After making cut 2, the cutaneous receptive field was mechanically stimulated again to confirm the unit was no longer activated. At least 3 min after cut 2, SA (firing rate, $>0.05 \mathrm{~Hz}$ persisting for $>5 \mathrm{~min}$ ) was recorded. The hindlimb was then stimulated once more to verify disconnection of the unit from its receptive field. Finally, cut 3 was made $3-4 \mathrm{~mm}$ central to the DRG, isolating the recorded segment of the DR. At least 3 min after cut 3 , SA was recorded for $5 \mathrm{~min}$. The hindlimb was stimulated a final time to confirm the continued absence of responses to peripheral stimulation. Conduction velocity $(\mathrm{CV})$ was determined after cut 3 by activating the $\mathrm{DR}$ with a stimulating electrode $(2 \mathrm{~ms}$ pulses, $0.5 \mathrm{~Hz}$ ) and measuring the latency of the action potential and the distance between the stimulating and recording electrodes $(8-15 \mathrm{~mm})$. DR fibers were classified by their CVs as $\mathrm{C}$ $(<1.2 \mathrm{~m} / \mathrm{s}), \mathrm{A} \delta(1.2-6.5 \mathrm{~m} / \mathrm{s})$, and $\mathrm{A} \alpha / \beta(>6.5 \mathrm{~m} / \mathrm{s})$ (Fang et al., 2005).

\section{Statistical analysis}

For all statistical analyses, values of $p<0.05$ were considered significant, and a sequential Bonferroni correction was applied to $p$ values involving multiple comparisons. All reported $p$ values are two-tailed. Analyses were performed with SAS 9.1, SPSS 16.0, and Prism 4.0 (GraphPad). 
Table 1. Electrophysiological properties of all small dissociated DRG neurons

\begin{tabular}{|c|c|c|c|c|c|c|}
\hline Property & Naive & Sham & $\mathrm{SCl}$ & SCl vs sham & SCl vs naive & Sham vs naive \\
\hline Soma diameter ( $\mu \mathrm{m})$ & $22.3 \pm 0.5(22,124)$ & $22.4 \pm 0.5(24,155)$ & $22.8 \pm 0.6(22,163)$ & NS & NS & NS \\
\hline Firing rate of neurons displaying SA (medians) $(\mathrm{Hz})$ & $1.0(12,19)$ & $1.3(15,25)$ & $1.0(23,72)$ & NS & NS & NS \\
\hline $\operatorname{RMP}(\mathrm{mV})$ & $-50.3 \pm 0.9(22,124)$ & $-49.3 \pm 0.7(24,157)$ & $-49.6 \pm 0.6(23,164)$ & NS & NS & NS \\
\hline Rheobase (400 ms pulse, pA) & $72.2 \pm 15.7(22,108)$ & $53.1 \pm 6.1(24,139)$ & $44.3 \pm 6.6(22,156)$ & NS & $p<0.01$ & $p<0.05$ \\
\hline AP threshold at $-50 \mathrm{mV}$ (2 ms pulse, $\mathrm{pA})$ & $366 \pm 33(21,114)$ & $365 \pm 29(24,148)$ & $298 \pm 23(22,141)$ & $p<0.01$ & $p<0.05$ & NS \\
\hline AP threshold at $-80 \mathrm{mV}$ (2 ms pulse, $\mathrm{pA})$ & $760 \pm 25(21,95)$ & $752 \pm 23(24,122)$ & $671 \pm 22(22,147)$ & $p<0.05$ & $p<0.05$ & NS \\
\hline Repetitive firing (spike number, 400 ms) & $2.9 \pm 0.3(22,116)$ & $3.0 \pm 0.3(24,145)$ & $3.7 \pm 0.3(23,150)$ & $p<0.05$ & $p<0.01$ & NS \\
\hline$R_{\mathrm{m}}(\mathrm{M} \Omega)$ & $551 \pm 31(21,117)$ & $591 \pm 39(24,151)$ & $693 \pm 44(22,161)$ & $p<0.05$ & $p<0.01$ & NS \\
\hline
\end{tabular}

Data are from both silent and SA neurons sampled from DRGs at all time points ( $\mathrm{d}$ to 8 months) and all levels (C6/C7, T8/T9, T11/T12, and L4/L5) from both male and female animals. Except in the case of firing rate of neurons displaying $S A$ (in which medians are presented), the numbers are means \pm SEM ( $N$ of animals, N of neurons). The $p$ values are for $t$ test (Bonferroni corrected) applied after overall significance was established with single-factor ANOVA. Firing rates were compared with the Kruskal-Wallis test and calculated as the total number of APs in the two $30 \mathrm{~s}$ observation periods divided by $60 \mathrm{~s}$.

In vitro electrophysiological data. All data from 456 neurons isolated from 69 animals were first screened (regardless of whether the corresponding animals had received behavioral tests) using factorial ANOVA to identify main effects and potential interactions on categorical variables. Treatment was thereby identified as the main effect on SA, and no significant interactions of treatment with time or with sex were found. Comparisons of gross frequencies of binary outcomes (presence or absence of SA) among treatment groups were made with Fisher's exact tests. For all continuous variables and proportionate data, we considered the individual rat to be the sampling unit (SU) (i.e., data points obtained from cells taken from the same individual were not considered independent observations). To avoid pseudoreplication and preserve the individual as the SU, we used nested ANOVA with post hoc $t$ tests (Bonferroni corrected) to compare electrophysiological variables among the treatment groups. SA recorded extracellularly in vivo was analyzed by singlefactor ANOVA followed by Newman-Keuls post hoc tests. Data that were not normally distributed (firing rates of SA neurons both in vivo and in vitro) were analyzed with Kruskal-Wallis or Mann-Whitney $U$ tests.

Behavioral data. To reduce the effects of baseline variability among animals, all behavioral data were expressed as differences from baseline. Changes in thermal sensitivity of the paws were analyzed using the mean for the three baseline tests from each paw subtracted from the mean from each paw taken after injury, and these four values (one from each paw) were averaged to form a grand mean of change in paw withdrawal latency for each rat. Changes in latency were compared among treatments using single-factor ANOVA with post hoc $t$ tests. Changes in mechanical sensitivity of the paws were made with nonparametric Kruskal-Wallis tests and Dunn's post hoc tests. Changes in mechanical sensitivity of the torso were analyzed by comparing the pooled incidence of all above-level and below-level responses using single-factor ANOVA with post hoc $t$ tests (Bonferroni corrected).

Correlation of SA with behavioral hypersensitivity. The proportion of dissociated DRG neurons from each tested level of the spinal cord exhibiting SA was averaged to obtain a single incidence of SA for each animal. These values were correlated with each rat's average change in latency for thermal sensitivity of the paws and with the change in mechanical sensitivity of the torso using the Pearson product-moment correlation. SA incidence per animal was correlated with each rat's change in threshold for mechanical sensitivity of the paws using Spearman's rank correlation. Linear regression was used to obtain a best-fit line for each plot shown in Figures 4 and 5 . However, because the $x$-axis represented a random rather than fixed variable, the $p$ values shown were derived from correlation rather than regression.

\section{Results}

SCI induces chronic SA that is generated by mechanisms intrinsic to small DRG neurons

The spinal contusion at T10 produced nearly complete hindlimb paralysis in all rats included in the study (SCI group's mean BBB score, $0.7 \pm 0.41 \mathrm{~d}$ after injury; $n=15$ ). Animals receiving sham surgery (sham group; $n=17$ ) exhibited no motor deficits (BBB score, $21.0 \pm 0.0$ ). Partial recovery of normal hindlimb motor function was observed 1 and 3-8 months after SCI (BBB score, $8.8 \pm 2.2$ and $13.7 \pm 2.1$, respectively; $n=5$ rats tested in each period), consistent with a moderate contusion injury (Grill, 2005; Carlton et al., 2009). No animals showed complete recovery after SCI.

To provide strong evidence that any SCI-induced alterations in sampled DRG neurons were intrinsic to those cells, small DRG neurons (Table 1) from animals in each group were dissociated and cultured at low density for 20-24 h before testing. Sampled neurons were never in direct contact with other cells (the nearest neighbor was $>100 \mu \mathrm{m}$ away) and were continuously superfused with fresh extracellular solution. We first asked whether SCI had a significant overall effect on SA when all the times of testing $(3 \mathrm{~d}$ to 8 months after injury) and spinal levels sampled (lumbar, thoracic, cervical) (see below) were combined (Fig. $1 A$ ). SA was observed in a larger proportion (43\%) of small DRG neurons dissociated from animals in the SCI group than from animals in the sham group $(16 \% ; p<0.0001)$ or naive group $(15 \%$; $p<$ 0.0001 ) (Fig. $1 B$ ). Although DRG neurons from animals with SCI were more likely to display SA, the firing rates observed when SA occurred in this group did not differ significantly from those seen when SA occurred in neurons from rats in the sham or naive groups (Table 1). The maximum firing rates (averaged across the $60 \mathrm{~s}$ observation period) were $5.6,11$, and $11 \mathrm{~Hz}$ in the naive, sham, and SCI groups, respectively. The SA patterns always appeared irregular (Figs. 1A, 3A).

The data on incidence of SA in dissociated DRG neurons after SCI were analyzed further to see whether this SA was more likely during acute or chronic phases after injury, or in neurons dissociated from particular spinal levels. No significant differences were found in the incidence of SA between neurons from the $3 \mathrm{~d}$ naive group and neurons from the 1-8 month naive group, so the naive data during these periods were combined to increase statistical power. Three days after injury (Fig. 1C), the incidence of SA was significantly increased in the SCI group compared with the sham $(p=0.04)$ and naive ( $p=0.0004$ ) groups in DRG neurons from L4/L5. Increased $\mathrm{SA}$ incidence relative to that in the naive group was also seen in neurons from T11/T12 ( $p=0.01)$, just below the T10 contusion site. However, SA incidence was not increased at this time by SCI in DRG neurons from T8/T9, just above the contusion site, or in neurons from $\mathrm{C} 6 / \mathrm{C} 7$, far above the contusion site.

One to 8 months after SCI (Fig. 1D), the same pattern of increased SA incidence was found in neurons from ganglia at L4/L5 ( $p=0.005$ and $p=0.03$, compared with sham and naive groups, respectively). Increased SA incidence relative to that in the naive group was also seen in neurons from T11/T12 ( $p=$ $0.04)$. A potentially important development was evident during this later phase immediately above the injury level (T8/T9), 
A Spontaneous activity (SA)

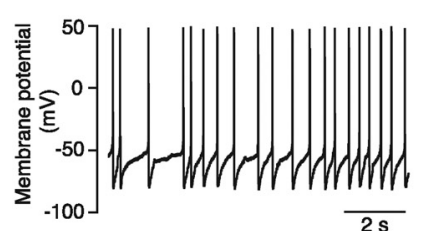

C
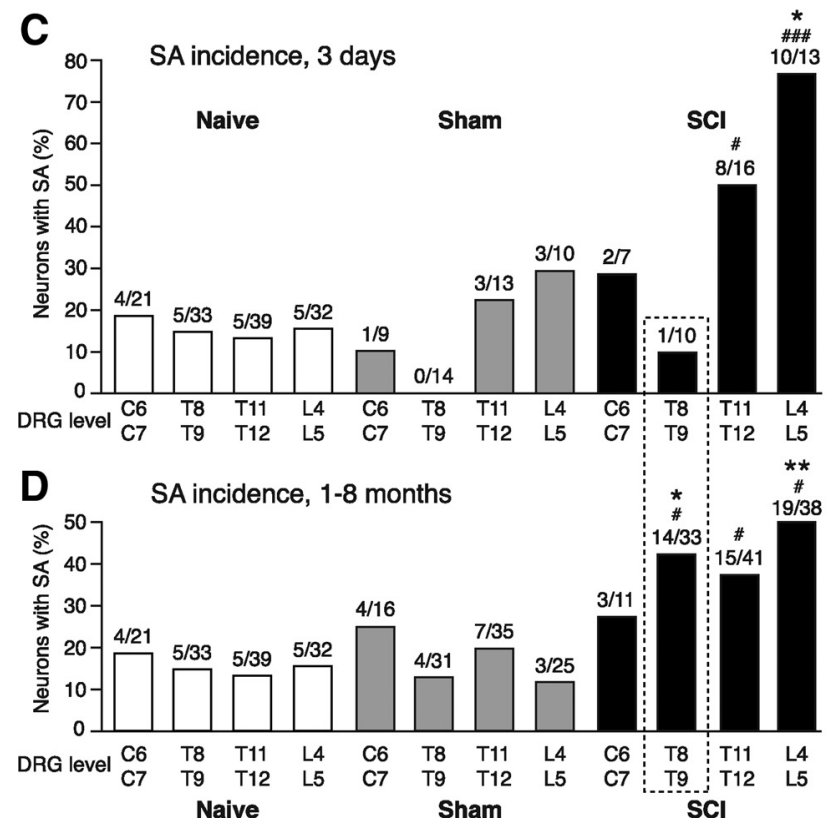

Figure 1. SCl increases the incidence of $S A$ in small DRG neurons recorded $1 \mathrm{~d}$ after dissociation. $\boldsymbol{A}$, Example of $S A(\sim 2 \mathrm{~Hz})$ in a DRG neuron dissociated from an $L 4 D R G 3$ d after $S C l$. $B$, Incidence of $S A$ in small DRG neurons dissociated from all sampled levels ( $(6, \mathrm{C} 7, \mathrm{~T} 8, \mathrm{~T} 9, \mathrm{~T} 11, \mathrm{~T} 12, \mathrm{~L} 4, \mathrm{~L} 5)$ and times after injury ( $3 \mathrm{~d}$ to 8 months). Ratios over each bar indicate the number of neurons exhibiting SA over the total number of neurons sampled in each group. The incidence of SA in the SCI group was significantly greater than that in the naive and sham groups. $C, D$, Incidence of $S A$ in dissociated DRG neurons at each sampled level $3 \mathrm{~d}$ after injury and $1-8$ months after injury. Within the naive group, no statistically significant differences were found overall or at any level between neurons tested $3 \mathrm{~d}$ and $1-8$ months after injury of the corresponding $\mathrm{SCl}$ and sham groups, so the naive data from these different test periods were combined to increase statistical power. The incidence of $S A$ was significantly greater in L4/L5 neurons in the SCl group (black) than in the naive (white) and sham (gray) groups 3 dand 1-8 months after injury, whereas in T8/T9 neurons SA was significantly elevated 1-8 months after SCI but not $3 \mathrm{~d}$ after $\mathrm{SCl}$ (dashed box). In this and subsequent figures, statistical significance is indicated as follows: SCl versus sham, ${ }^{*} p<0.05,{ }^{* *} p<0.01,{ }^{* * *} p<0.001$; SCl versus naive, ${ }^{\#} p<0.05,{ }^{\# \#} p<$ $0.01,{ }^{\# \#} p<0.001$.

where SA incidence was now significantly increased in the SCI group compared with the sham and naive groups $(p=0.01$ in each case) (see dashed box extending across Fig. $1 C, D)$. No other levels showed significant differences in SA incidence at 1-8 months compared with $3 \mathrm{~d}$ after injury. SA in DRG neurons above a moderate spinal contusion is more likely to excite intact pain pathways than SA in DRG neurons below the contusion level (which causes substantial interruption of ascending as well as descending axons) (for review, see Grill, 2005). No significant elevation of SA incidence was found in neurons from cervical ganglia (C6/C7), although the sample sizes at this level were relatively small. To examine the spatial pattern of SA incidence in the SCI group independent of the time of testing, SCI data was combined across the acute ( $3 \mathrm{~d}$ ) and more chronic (1-8 months) time periods; the incidence of SA after SCI increased from C6/7 (28\%), T8/9 (35\%), T11/12 (40\%), to L4/5 (57\%). When neurons from all spinal levels were compared across bins of cells tested at or near 3, 30, 90, 120, 180, and $240 \mathrm{~d}$ after injury, no significant differences were found in the incidence of SA across time in any group (naive, $p=0.62$; sham, $p=0.84$; $\mathrm{SCI}, p=0.69$ ).

To answer the overriding question of whether adult rats exhibit SCI-induced SA in small DRG neurons, both male and female rats were included in the in vitro study. SCI produced significant increases in the incidence of SA in both sexes, occurring in 43 of 84 DRG neurons dissociated from female rats after SCI $(51 \%)$, versus 9 of 49 neurons in the female sham group ( $18 \%)$, and 3 of 43 neurons in the female naive group $(7 \%)(p<$ 0.001 in each case). SA occurred in 29 of 85 DRG neurons dissociated from male rats after SCI (34\%), versus 16 of 104 neurons in the male sham group (15\%), and 16 of 82 neurons in the male naive group $(20 \%)(p=0.007$ and $p=0.05$, respectively). Although females showed a greater overall incidence of SA than did males after SCI ( $p=0.03$, Fisher's exact test), the interaction of injury with sex was not significant, suggesting a difference in the magnitude but not the direction of the injury effect between the sexes (factorial ANOVA; $p=0.52$ ). This study was not designed to assess sex differences; the extent and nature of differences between the sexes in SCI-induced SA in DRG neurons will be investigated in a separate study with sample sizes adequate to detect possible differences at specific levels and postinjury test times. Because SCI increased the overall incidence of SA in neurons from both male and female rats, data from male and female rats have been combined in this study to increase statistical power.

\section{Several manifestations of hyperexcitability are expressed in} spontaneously active but not silent DRG neurons after SCI We predicted that the intrinsic SA induced by SCI would be associated with neuronal hyperexcitability that would be manifest as enhanced responsiveness to depolarizing test stimulation, as has been observed in dissociated nociceptors after a form of chronic neuropathy induced near the spinal cord by compression of the DRG (Ma et al., 2005; Zheng et al., 2007). This prediction was confirmed when the electrophysiological properties of all neurons were compared across the naive, sham, and SCI groups (Table 1). Neurons in the SCI group required significantly lower currents to elicit APs during brief $2 \mathrm{~ms}$ pulses delivered at holding potentials of -50 or $-80 \mathrm{mV}$, and evoked greater repetitive firing during $400 \mathrm{~ms}$ pulses at $-50 \mathrm{mV}$. Rheobase (AP threshold during the long $400 \mathrm{~ms}$ pulse delivered at the $-50 \mathrm{mV}$ holding potential) was decreased relative to the naive group, and membrane resistance ( $R_{\mathrm{m}}$, measured under voltage clamp) was increased compared with neurons from rats in the sham and naive groups. No significant differences were observed in RMP or soma diameter (Table 1).

A surprising finding emerged when the effects of SCI were examined separately in electrically silent neurons and neurons exhibiting SA. The only statistically significant effect observed in silent neurons was a decrease in AP threshold at $-80 \mathrm{mV}$ compared with the naive group (Table 2). Similarly, when small DRG neurons exhibiting SA were examined by themselves, no significant differences among the naive, sham, and SCI groups were found (Table 3). These unexpected observations suggested that the major effect of SCI on small DRG neurons is to shift a large subset of these neurons into a hyperexcitable state that not only increases the responsiveness of these cells to depolarization but also causes most of the cells in this state to fire spontaneously (at least under our in vitro conditions). To test this interpretation, we compared the electrophysiological properties of all the silent neurons with all the neurons exhibiting SA (pooling across the naive, sham, and SCI groups) and found dramatic differences between 
Table 2. Electrophysiological properties of small dissociated DRG neurons lacking SA

\begin{tabular}{|c|c|c|c|c|c|c|}
\hline Property & Naive & Sham & $\mathrm{SCl}$ & SCl vs sham & SCl vs naive & Sham vs naive \\
\hline Soma diameter $(\mu \mathrm{m})$ & $23.1 \pm 0.4(22,106)$ & $22.8 \pm 0.3(24,126)$ & $22.9 \pm 0.4(22,96)$ & NS & NS & NS \\
\hline $\operatorname{RMP}(\mathrm{mV})$ & $-50.9 \pm 0.6(22,106)$ & $-50.8 \pm 0.6(24,128)$ & $-52.4 \pm 0.6(23,96)$ & NS & NS & NS \\
\hline Rheobase (400 ms pulse, pA) & $64.0 \pm 7.1(22,94)$ & $60.2 \pm 5.6(24,112)$ & $64.3 \pm 6.0(22,86)$ & NS & NS & NS \\
\hline AP threshold at $-50 \mathrm{mV}$ (2 ms pulse, $\mathrm{pA})$ & $383 \pm 21(21,96)$ & $378 \pm 19(24,121)$ & $367 \pm 25(22,81)$ & NS & NS & NS \\
\hline AP threshold at $-80 \mathrm{mV}$ (2 ms pulse, $\mathrm{pA}$ ) & $763 \pm 27(21,95)$ & $785 \pm 25(24,122)$ & $705 \pm 32(22,81)$ & NS & $p<0.05$ & NS \\
\hline Repetitive firing (spike number, 400 ms) & $2.8 \pm 0.2(22,102)$ & $2.8 \pm 0.3(24,122)$ & $2.9 \pm 0.2(23,94)$ & NS & NS & NS \\
\hline$R_{\mathrm{m}}(\mathrm{M} \Omega)$ & $534 \pm 28(21,99)$ & $541 \pm 28(24,121)$ & $592 \pm 38(22,95)$ & NS & NS & NS \\
\hline
\end{tabular}

Data are from silent neurons (no $S A$ ) sampled from DRGs at all time points and all spinal levels from male and female animals.

Table 3. Electrophysiological properties of small dissociated DRG neurons exhibiting SA

\begin{tabular}{|c|c|c|c|c|c|c|}
\hline Property & Naive & Sham & $\mathrm{SCl}$ & SCl vs sham & SCl vs naive & Sham vs naive \\
\hline Soma diameter ( $\mu \mathrm{m})$ & $19.4 \pm 0.8(12,19)$ & $20.8 \pm 0.8(15,25)$ & $21.8 \pm 0.5(22,71)$ & NS & NS & NS \\
\hline $\operatorname{RMP}(\mathrm{mV})$ & $-47.2 \pm 0.1(12,19)$ & $-47.2 \pm 1.0(15,25)$ & $-46.9 \pm 0.6(23,72)$ & NS & NS & NS \\
\hline Rheobase (400 ms pulse, pA) & $12.0 \pm 5.6(11,16)$ & $11.6 \pm 3.8(14,23)$ & $15.0 \pm 2.6(20,63)$ & NS & NS & NS \\
\hline AP threshold at $-50 \mathrm{mV}$ (2 ms pulse, $\mathrm{pA})$ & $164 \pm 40(12,19)$ & $185 \pm 40(14,23)$ & $180 \pm 19(21,64)$ & NS & NS & NS \\
\hline AP threshold at $-80 \mathrm{mV}$ (2 ms pulse, $\mathrm{pA}$ ) & $742 \pm 74(12,19)$ & $582 \pm 53(14,24)$ & $630 \pm 30(22,67)$ & NS & NS & NS \\
\hline Repetitive firing (spike number, 400 ms) & $4.1 \pm 0.6(11,16)$ & $3.6 \pm 0.7(13,19)$ & $4.5 \pm 0.4(23,60)$ & NS & NS & NS \\
\hline$R_{\mathrm{m}}(\mathrm{M} \Omega)$ & $786 \pm 118(12,19)$ & $911 \pm 86(15,24)$ & $932 \pm 50(22,70)$ & NS & NS & NS \\
\hline
\end{tabular}

Data are from neurons exhibiting SA, defined as at least one spontaneous AP during either of two $30 \mathrm{~s} \mathrm{sampling} \mathrm{periods} \mathrm{(}>3$ min after beginning the recording). Samples are from DRGs taken at all time points and all spinal levels from male and female animals.

properties of the silent and SA neurons. Some of these differences may be related to the association of SA with relatively depolarized RMP (Fig. 2A) and to the significantly more depolarized RMP of SA neurons (Fig. 2 B). In particular, links between SA and depolarized RMP might be associated with the profound differences between SA neurons and silent neurons in properties measured at a holding potential of $-50 \mathrm{mV}$ : rheobase (Fig. $2 C$ ), AP threshold during a brief depolarizing pulse (Fig. $2 D$ ), and repetitive firing during a long pulse (Fig. $2 F$ ). In addition, membrane resistance tested under voltage clamp with depolarizing pulses from -60 to $-55 \mathrm{mV}$ (a voltage range in which some SA occurred) (Fig. 2A) was significantly greater in SA neurons (Fig. $2 G$ ). Importantly, a marked difference was also found in a property measured at a hyperpolarized potential at which no SA was observed: AP threshold tested at a holding potential of $-80 \mathrm{mV}$ was significantly reduced in SA neurons (Fig. 2 E). These observations suggest that small DRG neurons can enter a hyperexcitable-SA state that is markedly different from the normal state. The hyperexcitable-SA state occurs infrequently in small DRG neurons from animals in the naive and sham groups, and its incidence is greatly increased by SCI.

\section{Many dissociated small DRG neurons display markers of nociceptors}

Most DRG neurons with soma diameters $<30 \mu \mathrm{m}$ in the L4/L5 DRGs are nociceptors (Lynn and Carpenter, 1982; Gold et al., 1996; Lawson, 2002; Fang et al., 2005), suggesting that many of the SA neurons we found in vitro were nociceptors. In separate experiments, we asked whether SA neurons from SCI animals display markers of two partially overlapping populations of nociceptors, sensitivity to capsaicin and binding of isolectin B4 (IB4 ${ }^{+}$cells) (Stucky and Lewin, 1999; Dirajlal et al., 2003). Neurons were sampled $3 \mathrm{~d}$ (nine cells), 1 month (five cells), and 3 months (eight cells) after SCI, and SA was observed (Fig. $3 A$ ) in 44,60 , and $63 \%$ of the neurons, respectively, replicating findings summarized in Figure 1. Large inward currents evoked by capsaicin (Fig. $3 B$ ) were observed in $83 \%$ of all the SA neurons. Moreover, $33 \%$ of the SA neurons were clearly $\mathrm{IB}^{+}{ }^{+}$(Fig. 3C,D), and $14 \%$ were both capsaicin sensitive and $\mathrm{IB}_{4}{ }^{+}$. Interestingly, none of the SA neurons sampled was neither capsaicin sensitive nor
$\mathrm{IB}^{+}$(i.e., all had one or both nociceptor markers). These results suggest that most of the dissociated neurons exhibiting SA $3 \mathrm{~d}$ to 3 months after SCI are nociceptors.

\section{SA in small DRG neurons is associated with pain-related behavioral alterations}

In a subset of the animals providing the data summarized in Figure 1, we tested changes in behavioral responsiveness caused by SCI $(n=$ 18 animals), sham treatment ( $n=18$ ), or no treatment (naive group; $n=14$ ), and calculated the incidence of SA per animal in small dissociated DRG neurons sampled from spinal levels relevant to the behavioral tests. Animals were tested between 1 and 5 months after injury, when hindlimb motor function had recovered sufficiently for the plantar surface of the hindpaw to be placed flush against the substrate (allowing comparable delivery of thermal and mechanical test stimuli across groups). To maximize the information we could extract about correlations of SA with behavioral alterations, we analyzed this data set for overall effects of injury on thermal sensitivity across all paws, mechanical sensitivity across all paws, and mechanical sensitivity across all tested regions of the torso, and then analyzed effects tested above and below the injury level separately (see next section). Confirming the results in Figure $1 B$, in which all neurons were compared (regardless of whether they were from animals that had received behavioral tests), SA incidence per animal was significantly greater in animals in the SCI group than in the sham or naive groups shortly after behavioral testing both 1 month (Fig. 4A) and 3-5 months (Fig. 4B) after injury. Consistent with previous behavioral findings (for review, see Vierck and Light, 2000; Hulsebosch et al., 2009; Yezierski, 2009), the SCI animals exhibited significant overall thermal hypersensitivity and mechanical hypersensitivity compared with sham and naive animals, both 1 month (Fig. 4C,E) and 3-5 months after injury (Fig. 4D, F). Hypersensitivity to these stimuli suggests that SCI produced mechanical allodynia and thermal hyperalgesia (Carlton et al., 2009). No significant differences were found between sham and naive groups, although the sham group showed a hint of mechanical hypersensitivity 3-5 months after surgery (Fig. $4 F$ ). Across all groups, there was a negative correlation between the overall incidence of SA and the latency to respond with hindpaw and forepaw withdrawal to a thermal test stimulus both 1 
A SA incidence and RMP
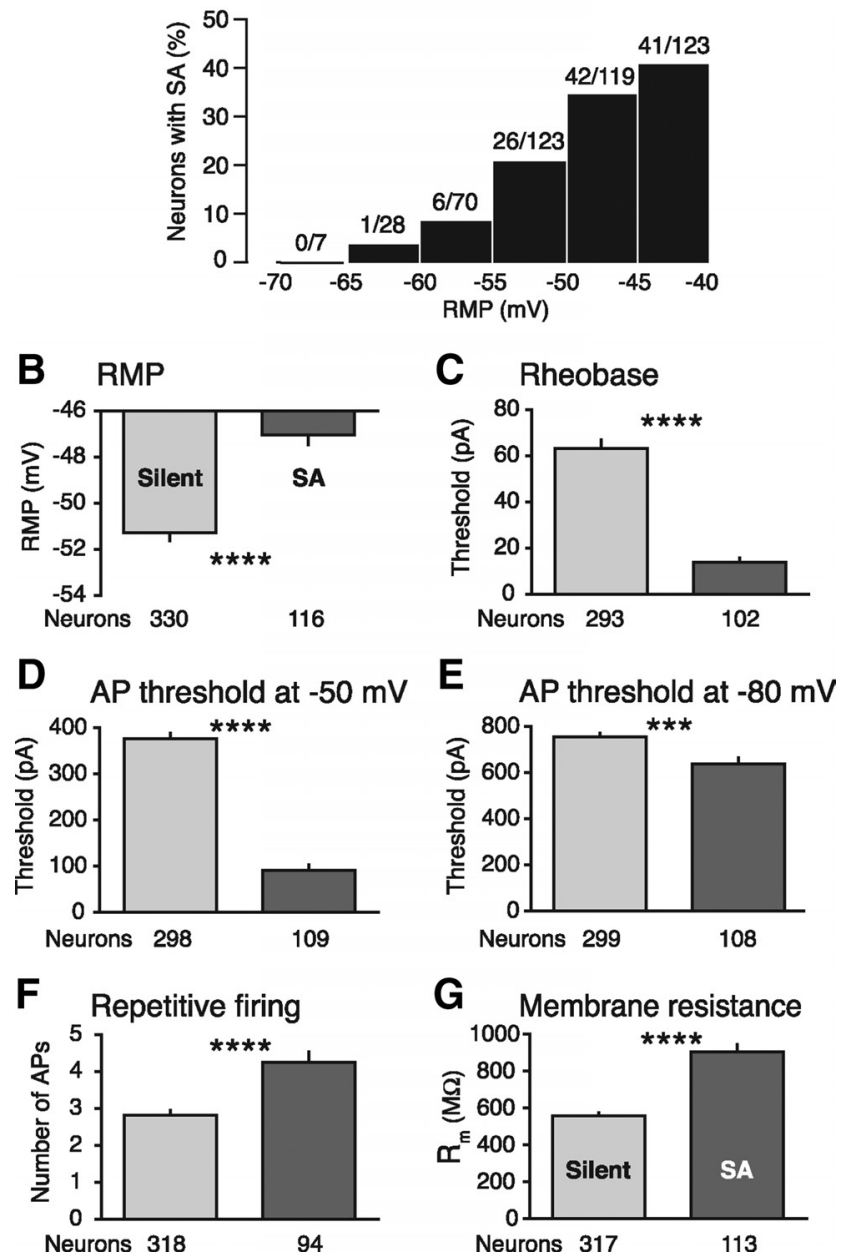

Figure 2. Electrophysiological differences between $S A$ neurons and silent neurons near RMP and at hyperpolarized holding potentials. $A$, Fractions of sampled neurons exhibiting $S A$ in different ranges of RMP. $\boldsymbol{B}$, Reduction of RMP in SA neurons. $\boldsymbol{C}$, Reduction of rheobase in SA neurons. Rheobase threshold was tested with $400 \mathrm{~ms}$ pulses delivered at a holding potential of $-50 \mathrm{mV}$. D, Reduction of AP threshold tested with $2 \mathrm{~ms}$ pulses at $-50 \mathrm{mV}$ in SA neurons. $\boldsymbol{E}$, Reduction of AP threshold tested with $2 \mathrm{~ms}$ pulses at $-80 \mathrm{mV}$ in $S A$ neurons. $\boldsymbol{F}$, Increase in repetitive firing tested with $400 \mathrm{~ms}$ pulses at $-50 \mathrm{mV}$. $G$, Increase in $R_{\mathrm{m}}$ tested under voltage clamp at $-60 \mathrm{mV}$. ${ }^{* *} p<0.001 ;{ }^{* * * *} p<0.0001$. Error bars indicate SEM.

and 3-5 months after injury (Fig. 4C,D). Significant negative correlation was also found between the incidence of SA and the mechanical threshold for hindpaw and forepaw withdrawal 1 and 3-5 months after injury (Fig. 4E,F). Furthermore, increased mechanical sensitivity of the torso was indicated by an increase in the combined incidence of vocalization, whole-body withdrawal, flinching, and orientation responses, which was found 3-5 months after SCI ( $n=$ 4) (data not shown; $p<0.01$ vs both naive and sham; $n=6$ and 7 , respectively). This torso hypersensitivity was positively correlated with increased SA ( $\left.p=0.008 ; r^{2}=0.62\right)$. A trend for torso hypersensitivity was seen 1 month after SCI, but this effect was not significant (data not shown) ( $p=0.08$, one-way ANOVA; $n=7,7$, and 5 in the naive, sham, and SCI groups, respectively). Together, the animals that exhibited the greatest incidence of SA in their DRG neurons after dissociation were also the animals that showed the greatest behavioral hypersensitivity to thermal and mechanical stimulation. As can be seen in the scatterplots (Fig. $4 C-F$ ), most of these animals were in the SCI group.
SA is correlated with behavioral changes below and above the injury level

Interruption of ascending and descending pathways by a spinal contusion would be expected to result in differences in painrelated responses elicited by stimuli below and above the level of the spinal lesion (Yezierski, 2009). We asked whether behavioral alterations expressed below and above the injury level are correlated with SA in populations of small DRG neurons dissociated, respectively, from DRGs below and above the spinal injury. SCI animals exhibited significant thermal hypersensitivity 1-5 months after injury compared with sham and naive animals when tests were delivered either to the hindpaws (Fig. 5A) or the forepaws (Fig. 5B). Shorter latency withdrawals of the hindpaws were correlated significantly with the incidence of SA in small DRG neurons dissociated from L4 and L5 ganglia (Fig. 5A). Although normal weight bearing by the hindlimb (Basso et al., $1995,1996)$ was often absent at 1 month (BBB score, $8.8 \pm 2.2$ ), test stimuli were only delivered when the plantar surface of the paw was flush against the glass. Nevertheless, the paw may not have been pressed as firmly against the glass in some SCI animals as in naive or sham animals. If so, this would be expected to reduce rather than enhance effective stimulus intensity in the SCI animals (increasing rather than decreasing withdrawal latency). Similar effects of spinal contusion injury on motor recovery and responses to hindpaw plantar stimulation have been observed in other studies (Mills et al., 2001), suggesting that possible differences in plantar pressure against the substrate neither account for nor prevent the expression of SCI-induced sensitization of hindlimb withdrawal responses after SCI with these stimuli at these time points.

In the case of the forepaws, too few cervical DRG neurons were sampled to allow meaningful assessment of correlations with behavior. However, assuming that SA in widespread abovelevel DRGs might contribute to spatially extensive central sensitization, we asked whether increased SA in neurons from all the above-level DRGs sampled (C6, C7, T8, T9) might be correlated with decreases in response latency of the forepaws to thermal stimulation. We found a significant correlation (Fig. 5B). Similar analyses were performed for mechanical hypersensitivity, which was significant when tests were delivered either to the hindpaws (Fig. 5C) or to the forepaws (Fig. 5D). Lower threshold withdrawals of the hindpaws were correlated significantly with higher incidence of SA in small DRG neurons dissociated from L4/L5 ganglia (Fig. 5C). Again, behavioral hypersensitivity (in this case, lower threshold withdrawals) of the forepaws was correlated significantly with higher incidence of SA in DRG neurons sampled from C6, C7, T8, and T9 (Fig. 5D), raising the possibility that SA in DRGs above the injury level but relatively distant from the forelimbs may contribute to sensitization of forelimb behavior after SCI. Again, no significant differences were found between sham and naive groups in behavioral responsiveness, although the sham group showed a hint of mechanical hypersensitivity in the forepaws (Fig. $5 D$ ). Also evident in Figure $5 D$ and Figures $4 C-F$ and $5 A-C$ is a consistent trend for behavioral response latencies and thresholds in naive control animals to increase $1-8$ months after their pretests. This change may reflect effects of age or possibly experience with the pretests, but it cannot be mistaken for the effects of injury reported here, which were in the opposite direction.

Unlike the hindlimb and forelimb withdrawal responses, which can be mediated by circuits within the spinal cord in the absence of supraspinal connections, vocalization requires supraspinal circuits. Consistent with (1) this difference in necessary circuitry and (2) substantial interruption of ascending fibers by the spinal contusion, no significant effect of SCI was found below 

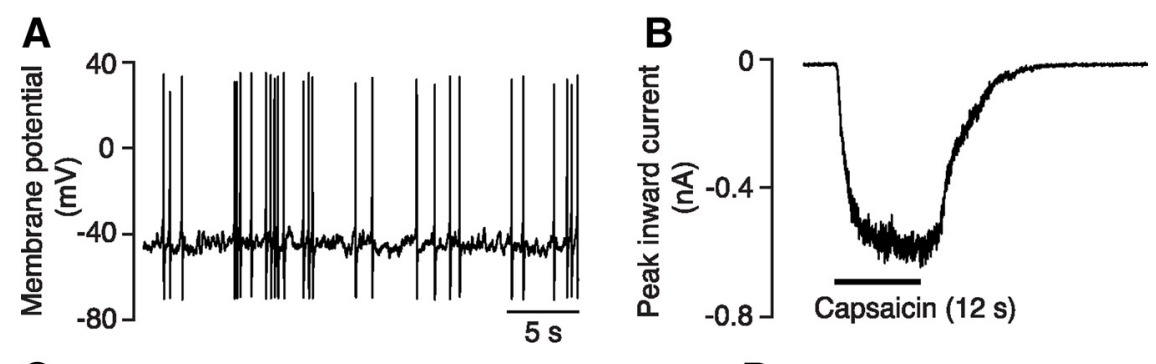

C

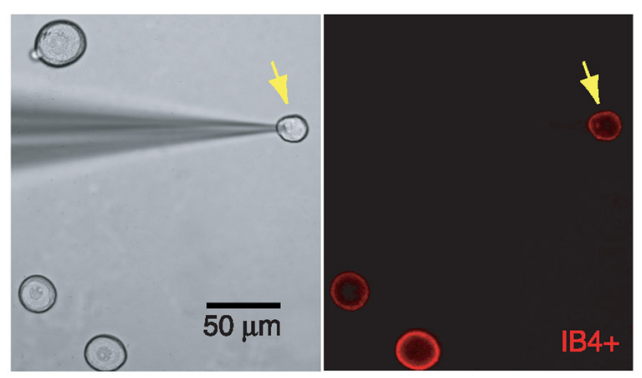

D

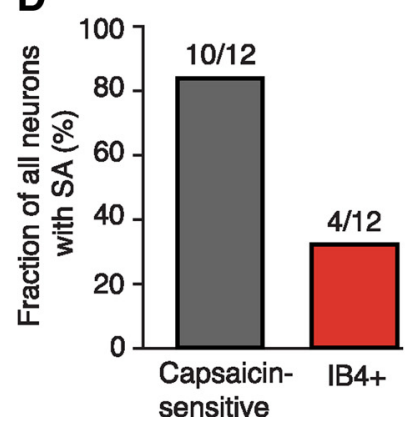

Figure 3. Dissociated small DRG neurons exhibit two markers for nociceptors. $A$, SA under current clamp in a neuron examined 3 months after SCI. B, Response of the same neuron to $3 \mu$ m capsaicin. $C$, Sampled neuron showing micropipette (left) and binding by fluorescent IB4 (right). Two other neurons were IB4 ${ }^{+}$, but the fourth (largest) neuron was not. D, Fractions of all neurons from $\mathrm{SCl}$ animals that exhibited SA and either responses to capsaicin or binding of isolectin B4 (IB4 ${ }^{+}$).

the injury level on the incidence of vocalization elicited by mechanical test stimuli delivered to the torso (Fig. $5 E$ ), and no significant correlation of SA incidence with vocalization incidence was found when SA at either L4/L5 (data not shown) or T11/T12 was considered (Fig. 5E). In contrast, above the injury level the incidence of vocalization in response to mechanical stimulation of the torso increased significantly in the SCI group compared with the sham and naive groups (Fig. $5 F$ ). The increased vocalization incidence was correlated significantly with increased SA in DRG neurons dissociated from C6, C7, T8, and T9 (Fig. 5F), suggesting that widespread SA in DRGs above the lesion contributes to the sensitization of this response. The increased vocalization after SCI elicited by above-level test stimuli shows that supraspinal circuits can be engaged under these conditions, and supports the possibility that the supraspinal circuits involved in the processing of allodynia, hyperalgesia, and spontaneous pain are also more easily activated in animals after SCI.

\section{SCI-induced SA is generated in C-and A $\delta$-fibers in or near the DRG in vivo}

The induction by SCI of an intrinsic hyperexcitable state in the somata of small dissociated DRG neurons that was correlated with behavioral hypersensitivity raised the possibility that SA might also be increased chronically in the somata of primary sensory neurons in vivo. We tested this possibility using anesthetized male rats in which lumbar DRs were surgically exposed so that DRG neuron activity could be recorded extracellularly from teased filaments (Fig. 6A). This transection of the DR central to the recording site (cut 1 ) also eliminated any recorded activity that might originate within the CNS. Thus, at the outset of recording the observed SA represented a combination of action potentials potentially initiated in the periphery, DRG, or DR. Disconnection of the DRG from the periphery (cut 2) revealed any SA generated in the proximal spinal nerve, DRG, and DR, and finally disconnection of the recording site from the DRG (cut 3 ) revealed any SA generated in the DR. Thus, SA that persisted after cut 2 and was eliminated by cut 3 had to be generated in or near the DRG. Examples of changes in gross SA recorded from filaments after cuts 2 and 3 are shown in Figure $6 B$.

Quantification of changes in SA was done at the level of individual sensory neurons, using template matching to extract single-unit activity (Fig. 6C) from the gross filament activity. To see whether SCI leads to generation of SA in or near the DRG, we examined units that exhibited SA before cut 2 and compared the proportions that still showed SA after cut 2. Note that we only examined units that showed SA to begin with; units that were silent in the absence of stimulation were not tested. Examples of unit activity patterns before and after cut 2 are shown in Figure $6 D$. Two of the examples (naive and sham 1) showed a complete abolition of SA by cut 2, whereas the sham 2 and SCI 1 examples showed a decrease in SA after cut 2. SCI 2 was a unit that displayed no obvious change in SA after cut 2 . This unit had an unusually high firing rate (see below). These examples illustrate the irregularity in firing pattern that characterized the SA observed in all single units in naive, sham, and SCI groups, and mirrors the irregularity in SA patterns seen in vitro (Figs. $1 A, 3 A$ ). Compared with the sham and naive groups, SCI resulted in a significantly higher incidence of singleunit SA remaining after cut 2 at each time point tested (Fig. $6 E$ ): $3 \mathrm{~d}(p<0.0001$ in each case $), 1$ month $(p=0.006$ and $p<$ 0.0001 , respectively), and 3 months ( $p=0.045$ and 0.03 , respectively) after injury. No SA was recorded in any units after cut 3. These results show that SCI promotes the generation of SA in (or near) the DRG in vivo. Because we did not examine units that were silent to begin with (to avoid additional sensitizing stimulation required for their identification), our in vivo experimental design did not reveal the number of units without SA before cut 2, and thus what the total incidence of SA was (i.e., SA generated in the DRG—probably in nociceptor somata-plus SA generated peripherally) in any of the groups. This also means that we could not distinguish a possible decrease in the incidence of somally generated SA over time (as might be suggested by the pattern in Fig. $6 E$ ) from the possibility of a progressive increase in the incidence of peripherally generated SA after SCI (Carlton et al., 2009). The latter possibility would increase the number of units exhibiting SA before cut 2 and thereby decrease the fraction retaining SA after cut 2 if the number with somally generated SA did not change over time.

In addition to the increase in incidence of SA generated in or near the DRG after SCI compared with the sham and naive groups, there was a modest but significant enhancement of SA incidence in the DRG 1 month (but not $3 \mathrm{~d}$ or 3 months) in the sham group compared with the naive group ( $p=0.03$ ) (Fig. $6 E$ ). No behavioral effects of sham treatment were seen at this or other time points (Fig. 4); however, the sample sizes in our behavioral study may not have been large enough to reveal any mild behavioral sensitization that might be related to modest increases in the incidence of nociceptor SA resulting from sham surgery.

In the units that continued to exhibit SA after cut 2, there was a tendency $3 \mathrm{~d}$ after injury for the firing rates to be higher in the SCI than sham group (medians, 3.0 vs $0.4 \mathrm{~Hz} ; n=59$ and 23, 
respectively; $p=0.10$, Mann-Whitney $U$ test). The median firing rate after cut 2 of the 14 single units in the naive group was $0.9 \mathrm{~Hz}$. However, no significant differences in firing rates after cut 2 were found between SCI and sham groups 1 and 3 months after injury (1 month medians, 1.6 vs $1.2 \mathrm{~Hz}$, respectively; 3 month medians, $0.4 \mathrm{~Hz}$ in each case). These firing rates were relatively close to those observed in vitro (Table 1). These results, like those from dissociated nociceptors, show that the effects of SCI on SA generated in the region of the soma were more prominent on the incidence of SA than on the firing rate during $\mathrm{SA}$. In each group, most of the firing rates after cut 2 were $<2 \mathrm{~Hz}$, but a few units in the SCI and sham groups had much higher rates; the maximum rates observed after cut 2 were $2.7,24.2$, and $55.7 \mathrm{~Hz}$ in the naive, sham, and SCI groups, respectively.

In 75 randomly selected single units in the SCI group, conduction velocities were measured to see whether the SA generated in or near the L4 and L5 DRGs after SCI occurred in sensory populations likely to contain nociceptors (i.e., sensory neurons with C- or A $\delta$-fibers) (Fang et al., 2005). SA continuing after cut 2 was observed in 44 of $57 \mathrm{C}$-fibers (77\%) and in 9 of $17 \mathrm{~A} \delta$-fibers (53\%). In this sample, only one $A \alpha / \beta$-fiber was found that exhibited SA before cut 2, and it failed to show SA after cut 2. These results indicate that $\mathrm{SCI}$ induces a persistent hyperexcitable state in $\mathrm{C}$ and $\mathrm{A} \delta$ sensory neurons that is expressed in vivo by an enhanced generation of SA in or near the somata of these neurons.

\section{Discussion}

This study has shown that SCI leads to a chronic hyperexcitablespontaneously active state in or near the somata of nociceptors, which is expressed both in vivo and in isolated DRG neurons, and which is correlated with behavioral indicators of pain.

\section{SCI promotes a chronic hyperexcitable-SA state that is expressed in nociceptor somata}

Examination of all dissociated DRG neurons in this study revealed that $43 \%$ of the SCI group exhibited SA versus only 16 and $15 \%$ in the sham and naive groups, respectively. SCI-induced SA was most prevalent in dissociated lumbar DRG neurons (57\%) and least prevalent in cervical DRG neurons (28\%), and the overall incidence of SA after SCI failed to decline for at least several months after injury. Because the DRG neurons were cultured at low density and exhibited SA $1 \mathrm{~d}$ after dissociation, the enhancement of SA incidence by SCI is likely to represent an intrinsic, long-lasting alteration of the soma. The nearest neighbor was $>100 \mu \mathrm{m}$ away, and continuous superfusion should have washed away secreted molecules, so any released factors would be present at extremely low concentrations compared with in vivo conditions, in which various cell types appose the somata or axons of DRG neurons, and in which the somata are exposed to injuryrelated factors both in the CSF and plasma (Abram et al., 2006).
B SA incidence per animal, 3-5 months

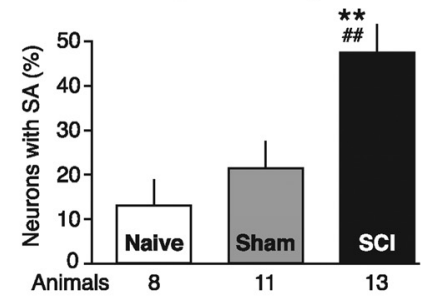

D Thermal hypersensitivity, 3-5 months
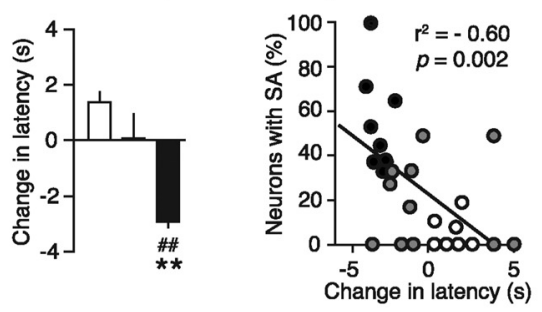

F Mechanical hypersensitivity, 3-5 months

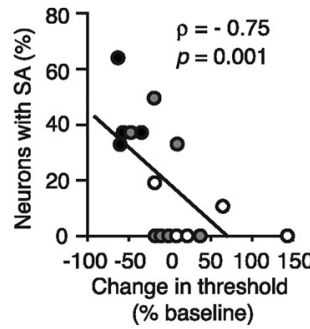

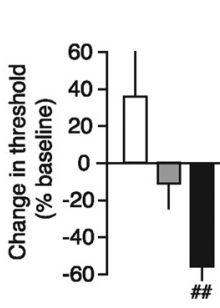

(\% baseline)

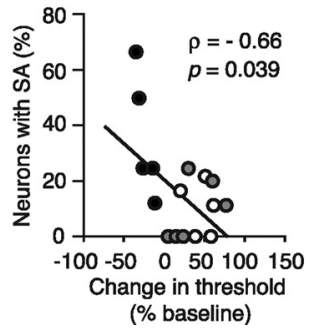

(\% baseline)

Figure 4. Increased $S A$ incidence after $S C$ is correlated with behavioral alterations. $A, B$, Significant enhancement of $S A$ incidence in small DRG neurons after $\mathrm{SCl}$ compared with the naive and sham groups when sampled 1 and 3-5 months after injury ( mechanical hypersensitivity (reduction in threshold for paw withdrawal) produced by $S C l$ is correlated significantly with increased SA 1 and 3-5 months after injury. White fill, Naive; gray fill, sham; black fill, SCI. Error bars indicate SEM.

Although a minority of the dissociated neurons had rudimentary neurites (usually shorter than the diameter of the soma) (S. S. Bedi, Q. Yang, E. T. Walters, unpublished observations), SA was found in neurons both without and with neurites, proving that at least some of the SA is generated within the soma. Nearly all of the small dissociated DRG neurons sampled after SCI and tested for nociceptor markers were capsaicin sensitive and/or bound IB4, consistent with reports that a large majority of small DRG neurons (soma diameter, $<30 \mu \mathrm{m}$ ) in the L4/L5 DRGs are nociceptors and have $\mathrm{C}$ - and $\mathrm{A} \delta$-fibers (Lynn and Carpenter, 1982; Gold et al., 1996; Lawson, 2002; Fang et al., 2005).

Recordings from dissociated neurons in vitro at room temperature showed that SCI promotes a persistent, widespread hyperexcitable state of nociceptors that is strongly linked to SA. Evidence that this state also results in SA generated in or near the somata of nociceptors in vivo at $37^{\circ} \mathrm{C}$ came from findings in anesthetized SCI animals of SA in single units of primary afferents at the L4/L5 level that often remained after disconnection from the periphery. Increased incidence of SA generated in the DRG occurred $3 \mathrm{~d}, 1$ month, and 3 months after SCI. Of the units tested for conduction velocity in the SCI group, $77 \%$ of the C-fibers exhibited SA in the isolated DRG, as did $53 \%$ of the A $\delta$-fibers, suggesting that many of the neurons displaying SA in vivo were nociceptors (Lawson, 2002). We do not yet know whether enhanced nociceptor SA occurs in vivo at additional spinal levels, as it does in vitro. 
A Thermal hypersensitivity, hindpaws
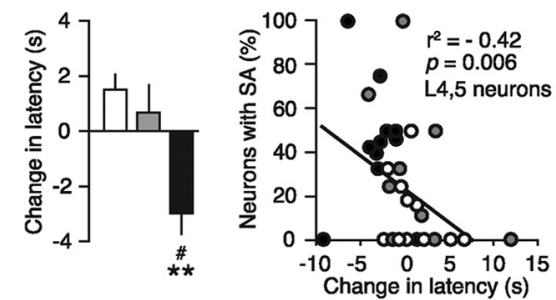

C Mechanical hypersensitivity, hindpaws
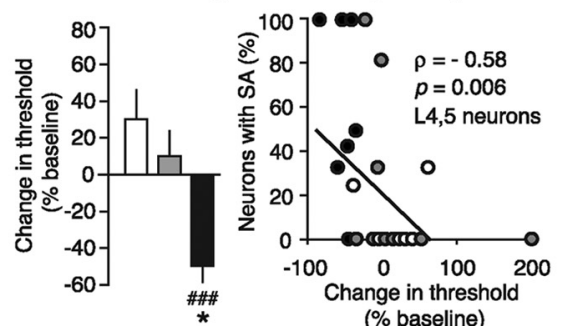

(\% baseline)

\section{E Vocalization, below injury level}
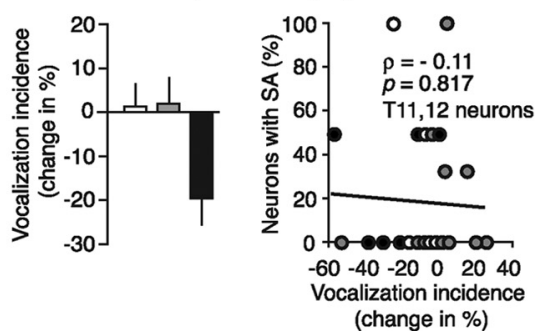
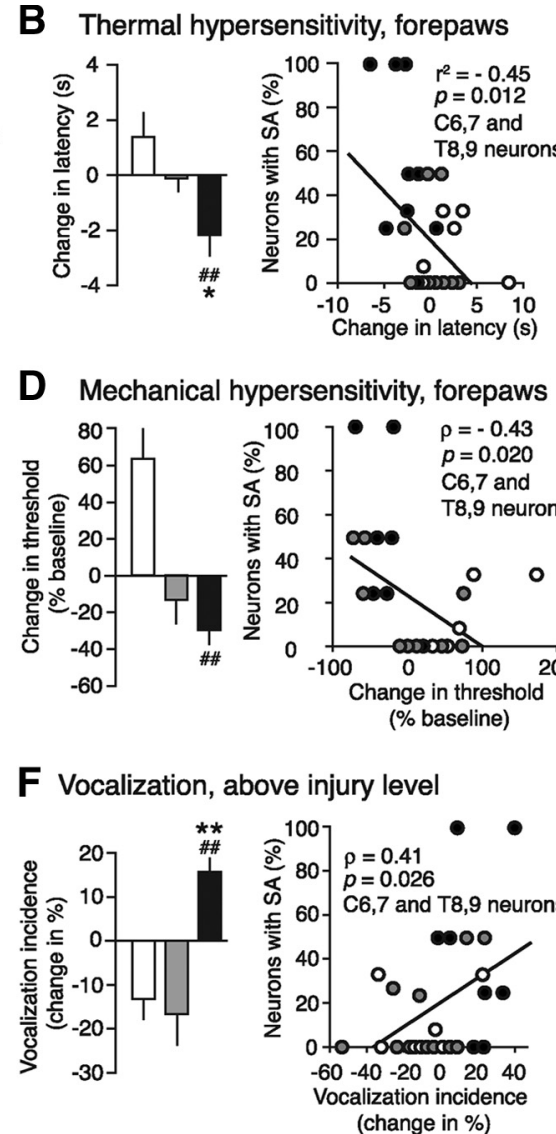

D Mechanical hypersensitivity, forepaws
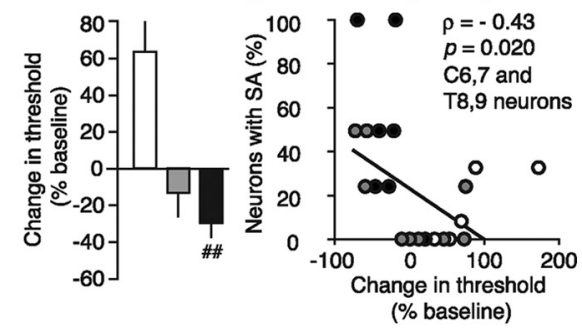

\section{F Vocalization, above injury level}

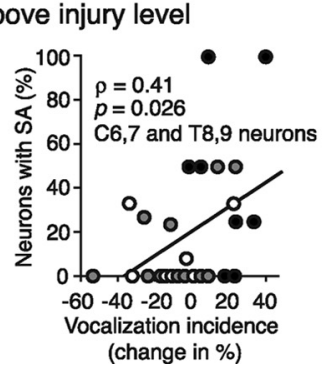

incidence in other studies (Ma and LaMotte, 2005). Indeed, most studies of dissociated nociceptors fail to mention SA, suggesting that nociceptor SA is rare under many experimental conditions. Our conditions differ from most whole-cell patch studies of dissociated nociceptors by testing $1 \mathrm{~d}$ after dissociation, rather than acutely, and (compared with other longer-term studies) by omitting any serum or growth factors to minimize neurite outgrowth. Either of these conditions might enhance entry into the hyperexcitable-SA state. In vivo, we had no difficulty finding SA units in naive animals, but we cannot compare the incidence of SA in these experiments with those reported by others because we did not determine the number of silent units. Although nociceptors are usually silent in vivo, ongoing background activity does occur. For example, under control, in vivo conditions, the incidence of nociceptor SA has been reported as 7\% (Xie et al., 2005), 9\% (Djouhri et al., 2006), and 13\% (Xu and Brennan, 2010). If the incidence of C-fiber SA in our naive animals was $\sim 10 \%$ and each teased filament contained 5-10 C-fiber units, many of the sampled filaments would have had at least one SA C-fiber.

\section{SA generated in nociceptors may} contribute to chronic pain after SCI Confirming previous findings (for review, see Vierck and Light, 2000; Hulsebosch et al., 2009; Yezierski, 2009), animals with SCI exhibited thermal and mechanical hypersensitivity both below and above the level of the contusion 1 and 3-5 months after injury. Importantly, animals displaying the greatest behavioral responsiveness to mechanical and thermal test stimuli

Dissociated nociceptors also displayed other alterations that increase excitability after SCI, including a decrease in RMP, decreases in rheobase and other measures of AP threshold, and increases in repetitive firing and membrane resistance. Surprisingly, these properties showed virtually no significant alterations in electrically silent neurons after SCI-all of the differences in excitability properties between the SCI group and the two control groups were accounted for by the alterations in the SA neurons. Moreover, enormous differences were found in excitability properties between silent and SA neurons in every group, but these properties in SA neurons did not differ significantly between the SCI group and control groups. These results indicate that SCI greatly facilitates the entry of nociceptors into a chronic hyperexcitable-SA state that occurs infrequently under the conditions experienced by our naive and sham groups. An interesting question is whether this nociceptor state, in which profound hyperexcitability is coupled with a strong propensity to fire spontaneously, also contributes to other forms of chronic pain.

The 15-16\% overall incidence of in vitro SA in our two control groups is similar to the $13 \%$ incidence of acute in vitro SA after sham surgery found by Zheng et al. (2007) but higher than the SA also displayed the greatest incidence of SA in dissociated nociceptors. Although this does not prove that nociceptor SA helps to drive allodynia and hyperalgesia after SCI, two considerations support this possibility. First, activity in nociceptors excites pain pathways and drives central sensitization, amplifying central activity that produces pain, allodynia, and hyperalgesia (Woolf, 2007; Woolf and Ma, 2007). Interestingly, Djouhri et al. (2006) reported that spontaneous pain in rats is produced during peripheral inflammation when there is a high incidence $(30-60 \%)$ of nociceptors displaying SA at relatively low individual firing rates $(0.5-2 \mathrm{~Hz})$. The SA incidence $(\sim 40-75 \%)$ and median firing rates ( $1.0 \mathrm{~Hz}$ in vitro and $0.4-3.0 \mathrm{~Hz}$ in vivo) we observed after SCI were in the same range. Second, elevated SA in vitro occurred by 1 month after SCI in DRG neurons sampled from T8 and T9-above the spinal contusion site. If SA also occurs in vivo in nociceptors above the lesion, this continuing activity- unlike SA below the lesion-should have uninterrupted access to intact pain pathways, potentially driving conscious at-level and abovelevel pain. Unlike "clinically incomplete" spinal injuries that spare many ascending axons (Detloff et al., 2008; Hall et al., 2010), our behavioral data suggest that limited communication 
occurred across the contusion site. Specifically, the effects on torso-elicited vocalization differed dramatically when test stimuli were delivered above and below the contusion level (Fig. 5E,F), although a sufficient number of spared axons may have remained to enable perception of below-level pain. An interesting finding was that hypersensitivity of withdrawal responses of the hindlimbs was at least as great as that of the forelimbs after SCI (Fig. 5). This is consistent with these flexor responses being mediated by local spinal circuits, with possible facilitation caused by loss of descending inhibitory influences after SCI (Andersen et al., 2004). The delayed emergence of SA in neurons sampled from DRGs above the injury level (correlated with increased vocalization) is interesting because it suggests a potential parallel to the slow development of chronic pain in SCI patients (Siddall et al., 2003; Cruz-Almeida et al., 2009). Widespread SA and hyperexcitability occurring in smaller DRG neurons would also be expected to contribute to other problems after SCI, including autonomic dysreflexia (Krenz et al., 1999; Black et al., 2003; de Groat and Yoshimura, 2010).

Possible causes of the nociceptor hyperexcitable-SA state after SCI In principle, one cause of SCI-induced SA might be direct injury of central processes of nociceptors. Axotomy of DRG neurons can promote SA (Burchiel, 1984; Amir and Devor, 1993; Liu et al., 2000). Axotomizing injury caused by dissociation might explain the somewhat higher incidence of SA we found in dissociated DRG neurons in the naive group $(\sim 15 \%)$ than has been reported in more intact DRG preparations ( $\sim 0 \%)$ (Zheng et al., 2007). Axotomy-induced SA cannot, however, explain the pattern of SA observed after SCI. Less SA occurred in DRG neurons taken from T11/T12, immediately below the SCI level than from L4/L5 DRGs seven segments away, and a high incidence of SA generated in the DRG also occurred at $\mathrm{L} 4 / \mathrm{L} 5$ in vivo. Although a few $\mathrm{C}$ - and $\mathrm{A} \delta$ fibers project up to seven segments from their segment of entry (Traub et al., 1990; Lidierth, 2007), most project only one to two segments (Chung et al., 1979). Interestingly, expression of ATF3 (activating transcription factor-3) (a cellular stress marker inducible by axotomy) is reportedly absent in DRGs distant from a site of SCI (in this case, cervical DRGs), but abundant in DRGs neighboring the lesion (Carlton et al., 2009) (see also Huang et al., 2006). The greatest incidence of SA occurred in dissociated nociceptors taken below

A

F

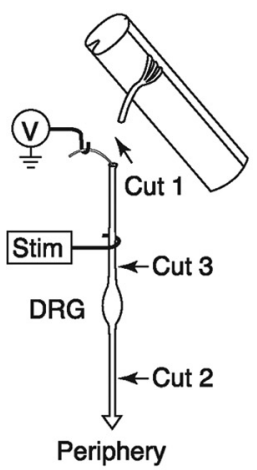

B Gross SA recorded from filament

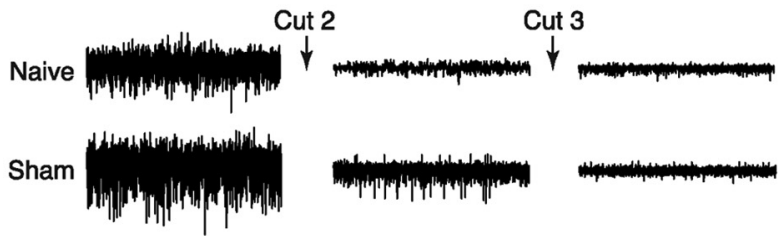

$\mathrm{scl}$
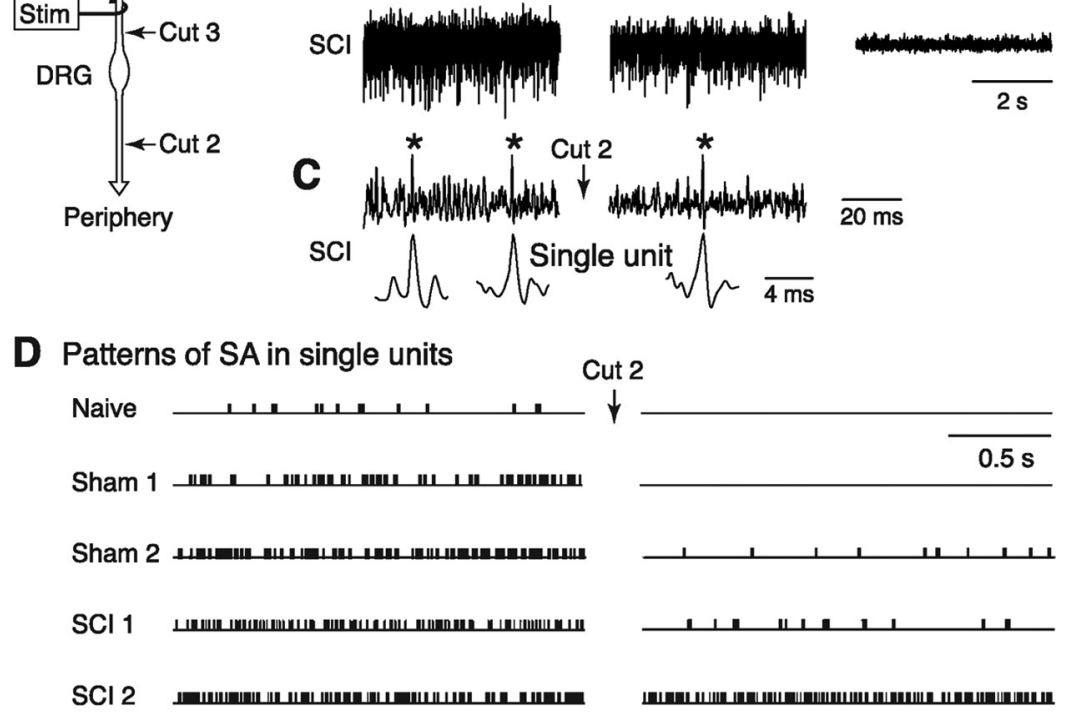

E Fraction of single units with SA that retain SA after Cut 2
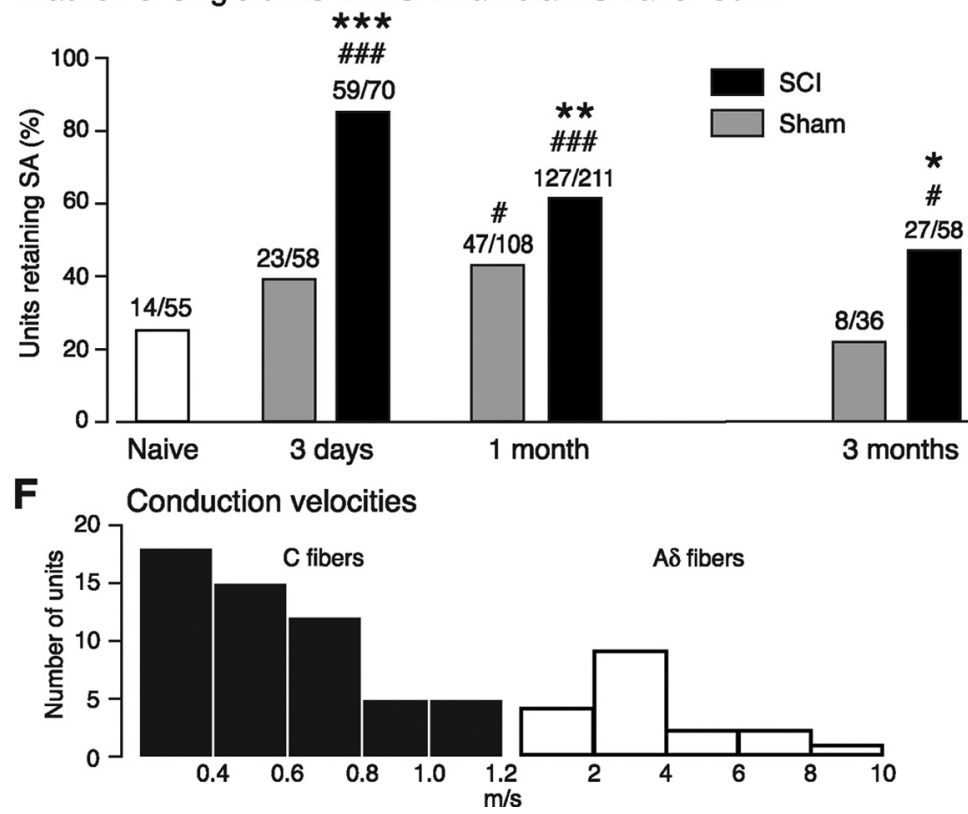

Figure 6. Increased incidence of SA occurs in vivo after SCl and is generated in or near the DRG. $A$, Schematic showing the in vivo site for recording dorsal root filaments and sites of transection used to demonstrate activity generated in or near the DRG. $\boldsymbol{B}_{\text {, }}$ Examples of gross activity recorded extracellularly from dorsal root filaments. $C$, Example of an action potential identified with a single unit before and after cut 2 in an $\mathrm{SCl}$ animal. $\boldsymbol{D}$, Examples of patterns of single-unit activity before and after cut 2 . The naive and sham 1 examples showed complete abolition of $S A$ by cut 2 . Sham 2 and SCI 1 showed a reduction in frequency after cut 2 (to $\sim 3$ and $\sim 4 \mathrm{~Hz}$, respectively). SCI 2 showed no change after cut 2 (remaining at $\sim 30 \mathrm{~Hz}$ ). $\boldsymbol{E}$, Increased incidence of single-unit $S A$ after cut 2 (generated in or near the DRG) after $\mathrm{SCl}$ at different times after injury. Indicated $p$ values compare the incidence of remaining $S A$ in the $S C I$ group to $S A$ in the corresponding sham group at the same time point and to $S A$ in the single naive group. $\boldsymbol{F}$, Distribution of conduction velocities randomly selected for measurement in a subset of the tested units.

the injury site, most prominently in lumbar nociceptors but also in nociceptors immediately below the lesion, which may suggest a role for interrupted descending influences (e.g., disinhibition) in induction of the hyperexcitable-SA state. Three 
days after injury, the lowest incidence of SA occurred in nociceptors immediately above the injury, suggesting that transient effects of SCI or the associated surgery might briefly oppose the induction of this state locally. However, during the chronic phase, this state was also present in nociceptors above the lesion site, where it might contribute to above-level pain. The lack of apparent SCI-induced SA in somata of cervical nociceptors is unexpected because SCI enhances SA in peripheral fibers of these nociceptors (Carlton et al., 2009). It will be interesting to see whether SCI also enhances peripherally generated SA at other levels and how closely the somal hyperexcitable-SA state is linked to enhancement of peripherally generated SA.

Another cause of chronic nociceptor SA is suggested by findings of persistently activated microglia and astroglia in the dorsal horn of lumbar (Nesic et al., 2005; Hains and Waxman, 2006; Detloff et al., 2008; Gwak and Hulsebosch, 2009) and cervical (Carlton et al., 2009) segments distant from a site of thoracic SCI. The primary targets of cytokines, chemokines, $\mathrm{PGE}_{2}$, and other factors released from activated glia after spinal cord injury are often assumed to be dorsal horn neurons (Zhao et al., 2007; Detloff et al., 2008), but these same factors may act on central processes of nociceptors to trigger nociceptor hyperexcitability and SA (Miller et al., 2009). Similar factors are released from macrophages and other inflammatory cells that infiltrate into the spinal cord after SCI (Beck et al., 2010), and into DRGs distant from the spinal lesion (McKay and McLachlan, 2004). Although the nociceptor soma may not be a major site for generating SA during peripheral inflammation (Katz and Gold, 2006), inflammatory signals released more centrally, or directly into DRGs, after SCI may trigger SA generation within the soma.

SA occurring in nociceptor somata would be expected to complement and might drive the peripheral sensitization that follows SCI (Carlton et al., 2009), and enhanced activity in nociceptors resulting from both increased peripheral sensitivity and spontaneous spike generation (in the soma and periphery) might contribute significantly to pain after SCI. Prolonged SA is a necessary early trigger of persistent pain in two peripheral neuropathic pain models (Xie et al., 2005). Interestingly, early blockade of primary afferent SA in these models also reduces microglial and astrocytic activation (Xie et al., 2009). This suggests that a positive-feedback relationship might drive chronic pain after SCI (and possibly other central neuropathies), with glial activation triggering a hyperexcitable-SA state in nociceptors, and the resulting activity feeding back to maintain glial activation, resensitization (central and peripheral) of the nociceptors, and pain. Such interactions would blur widely accepted distinctions between neuropathic pain and nociceptive pain, and between central sensitization and peripheral sensitization, and encourage the search for therapeutically promising strategies to block SA in nociceptors.

\section{References}

Abram SE, Yi J, Fuchs A, Hogan QH (2006) Permeability of injured and intact peripheral nerves and dorsal root ganglia. Anesthesiology 105:146-153.

Ali Z, Ringkamp M, Hartke TV, Chien HF, Flavahan NA, Campbell JN, Meyer RA (1999) Uninjured C-fiber nociceptors develop spontaneous activity and alpha-adrenergic sensitivity following L6 spinal nerve ligation in monkey. J Neurophysiol 81:455-466.

Amir R, Devor M (1993) Ongoing activity in neuroma afferents bearing retrograde sprouts. Brain Res 630:283-288.

Andersen OK, Finnerup NB, Spaich EG, Jensen TS, Arendt-Nielsen L (2004) Expansion of nociceptive withdrawal reflex receptive fields in spinal cord injured humans. Clin Neurophysiol 115:2798-2810.

Basso DM, Beattie MS, Bresnahan JC (1995) A sensitive and reliable locomotor rating scale for open field testing in rats. J Neurotrauma 12:1-21.
Basso DM, Beattie MS, Bresnahan JC (1996) Graded histological and locomotor outcomes after spinal cord contusion using the NYU weight-drop device versus transection. Exp Neurol 139:244-256.

Beck KD, Nguyen HX, Galvan MD, Salazar DL, Woodruff TM, Anderson AJ (2010) Quantitative analysis of cellular inflammation after traumatic spinal cord injury: evidence for a multiphasic inflammatory response in the acute to chronic environment. Brain 133:433-447.

Black JA, Cummins TR, Yoshimura N, de Groat WC, Waxman SG (2003) Tetrodotoxin-resistant sodium channels $\mathrm{Na}_{\mathrm{v}} 1.8 / \mathrm{SNS}$ and $\mathrm{Na}_{\mathrm{v}} 1.9 / \mathrm{NaN}$ in afferent neurons innervating urinary bladder in control and spinal cord injured rats. Brain Res 963:132-138.

Bove GM, Dilley A (2010) The conundrum of sensitization when recording from nociceptors. J Neurosci Methods 188:213-218.

Bove GM, Ransil BJ, Lin HC, Leem JG (2003) Inflammation induces ectopic mechanical sensitivity in axons of nociceptors innervating deep tissues. J Neurophysiol 90:1949-1955.

Burchiel KJ (1984) Effects of electrical and mechanical stimulation on two foci of spontaneous activity which develop in primary afferent neurons after peripheral axotomy. Pain 18:249-265.

Burchiel KJ, Russell LC, Lee RP, Sima AA (1985) Spontaneous activity of primary afferent neurons in diabetic BB/Wistar rats. A possible mechanism of chronic diabetic neuropathic pain. Diabetes 34:1210-1213.

Carlton SM, Du J, Tan HY, Nesic O, Hargett GL, Bopp AC, Yamani A, Lin Q, Willis WD, Hulsebosch CE (2009) Peripheral and central sensitization in remote spinal cord regions contribute to central neuropathic pain after spinal cord injury. Pain 147:265-276.

Caterina MJ, Leffler A, Malmberg AB, Martin WJ, Trafton J, Petersen-Zeitz KR, Koltzenburg M, Basbaum AI, Julius D (2000) Impaired nociception and pain sensation in mice lacking the capsaicin receptor. Science 288:306-313.

Chaplan SR, Bach FW, Pogrel JW, Chung JM, Yaksh TL (1994) Quantitative assessment of tactile allodynia in the rat paw. J Neurosci Methods 53:55-63.

Chung K, Langford LA, Applebaum AE, Coggeshall RE (1979) Primary afferent fibers in the tract of Lissauer in the rat. J Comp Neurol 184:587-598.

Crown ED, Ye Z, Johnson KM, Xu GY, McAdoo DJ, Hulsebosch CE (2006) Increases in the activated forms of ERK 1/2, p38 MAPK, and CREB are correlated with the expression of at-level mechanical allodynia following spinal cord injury. Exp Neurol 199:397-407.

Cruz-Almeida Y, Felix ER, Martinez-Arizala A, Widerström-Noga EG (2009) Pain symptom profiles in persons with spinal cord injury. Pain Med 10:1246-1259.

Cruz-Orengo L, Figueroa JD, Velázquez I, Torrado A, Ortíz C, Hernández C, Puig A, Segarra AC, Whittemore SR, Miranda JD (2006) Blocking EphA4 upregulation after spinal cord injury results in enhanced chronic pain. Exp Neurol 202:421-433.

Dang K, Bielfeldt K, Lamb K, Gebhart GF (2005) Gastric ulcers evoke hyperexcitability and enhance $\mathrm{P} 2 \mathrm{X}$ receptor function in rat gastric sensory neurons. J Neurophysiol 93:3112-3119.

de Groat WC, Yoshimura N (2010) Changes in afferent activity after spinal cord injury. Neurourol Urodyn 29:63-76.

Detloff MR, Fisher LC, McGaughy V, Longbrake EE, Popovich PG, Basso DM (2008) Remote activation of microglia and pro-inflammatory cytokines predict the onset and severity of below-level neuropathic pain after spinal cord injury in rats. Exp Neurol 212:337-347.

Dirajlal S, Pauers LE, Stucky CL (2003) Differential response properties of $\mathrm{IB}_{4}$-positive and -negative unmyelinated sensory neurons to protons and capsaicin. J Neurophysiol 89:513-524.

Dixon WJ (1991) Staircase bioassay: the up-and-down method. Neurosci Biobehav Rev 15:47-50.

Djouhri L, Dawbarn D, Robertson A, Newton R, Lawson SN (2001) Time course and nerve growth factor dependence of inflammation-induced alterations in electrophysiological membrane properties in nociceptive primary afferent neurons. J Neurosci 21:8722-8733.

Djouhri L, Koutsikou S, Fang X, McMullan S, Lawson SN (2006) Spontaneous pain, both neuropathic and inflammatory, is related to frequency of spontaneous firing in intact C-fiber nociceptors. J Neurosci 26:12811292.

Du J, Zhou S, Coggeshall RE, Carlton SM (2003) N-methyl-D-aspartateinduced excitation and sensitization of normal and inflamed nociceptors. Neuroscience 118:547-562. 
Fang X, McMullan S, Lawson SN, Djouhri L (2005) Electrophysiological differences between nociceptive and non-nociceptive dorsal root ganglion neurones in the rat in vivo. J Physiol 565:927-943.

Finnerup NB, Jensen TS (2004) Spinal cord injury pain-mechanisms and treatment. Eur J Neurol 11:73-82.

Gold MS, Dastmalchi S, Levine JD (1996) Co-expression of nociceptor properties in dorsal root ganglion neurons from the adult rat in vitro. Neuroscience 71:265-275.

Gracely RH, Lynch SA, Bennett GJ (1992) Painful neuropathy: altered central processing maintained dynamically by peripheral input. Pain 51:175-194.

Greffrath W, Schwarz ST, Büsselberg D, Treede RD (2009) Heat-induced action potential discharges in nociceptive primary sensory neurons of rats. J Neurophysiol 102:424-436.

Grill RJ (2005) User-defined variables that affect outcome in spinal cord contusion/compression models. Exp Neurol 196:1-5.

Gwak YS, Hulsebosch CE (2009) Remote astrocytic and microglial activation modulates neuronal hyperexcitability and below-level neuropathic pain after spinal injury in rat. Neuroscience 161:895-903.

Hains BC, Waxman SG (2006) Activated microglia contribute to the maintenance of chronic pain after spinal cord injury. J Neurosci 26: $4308-4317$

Hall BJ, Lally JE, Vukmanic EV, Armstrong JE, Fell JD, Gupta DS, Hubscher $\mathrm{CH}$ (2010) Spinal cord injuries containing asymmetrical damage in the ventrolateral funiculus is associated with a higher incidence of at-level allodynia. J Pain 11:864-875.

Hargreaves K, Dubner R, Brown F, Flores C, Joris J (1988) A new and sensitive method for measuring thermal nociception in cutaneous hyperalgesia. Pain 32:77-88.

Huang WL, Robson D, Liu MC, King VR, Averill S, Shortland PJ, Priestley JV (2006) Spinal cord compression and dorsal root injury cause upregulation of activating transcription factor-3 in large-diameter dorsal root ganglion neurons. Eur J Neurosci 23:273-278.

Hulsebosch CE, Hains BC, Crown ED, Carlton SM (2009) Mechanisms of chronic central neuropathic pain after spinal cord injury. Brain Res Rev 60:202-213.

Kajander KC, Bennett GJ (1992) Onset of a painful peripheral neuropathy in rat: a partial and differential deafferentation and spontaneous discharge in A beta and A delta primary afferent neurons. J Neurophysiol 68:734-744.

Katz EJ, Gold MS (2006) Inflammatory hyperalgesia: a role for the C-fiber sensory neuron cell body? J Pain 7:170-178.

Koerber HR, Druzinsky RE, Mendell LM (1988) Properties of somata of spinal dorsal root ganglion cells differ according to peripheral receptor innervated. J Neurophysiol 60:1584-1596.

Koltzenburg M, Bennett DL, Shelton DL, McMahon SB (1999) Neutralization of endogenous NGF prevents the sensitization of nociceptors supplying inflamed skin. Eur J Neurosci 11:1698-1704.

Krenz NR, Meakin SO, Krassioukov AV, Weaver LC (1999) Neutralizing intraspinal nerve growth factor blocks autonomic dysreflexia caused by spinal cord injury. J Neurosci 19:7405-7414.

Lawson SN (2002) Phenotype and function of somatic primary afferent nociceptive neurones with C-, Adelta- or Aalpha/beta-fibres. Exp Physiol 87:239-244.

Lidierth M (2007) Long-range projections of Adelta primary afferents in the Lissauer tract of the rat. Neurosci Lett 425:126-130.

Liu X, Eschenfelder S, Blenk KH, Jänig W, Häbler H (2000) Spontaneous activity of axotomized afferent neurons after L5 spinal nerve injury in rats. Pain 84:309-318.

Lynn B, Carpenter SE (1982) Primary afferent units from the hairy skin of the rat hind limb. Brain Res 238:29-43.

Ma C, LaMotte RH (2005) Enhanced excitability of dissociated primary sensory neurons after chronic compression of the dorsal root ganglion in the rat. Pain 113:106-112.

McKay SM, McLachlan EM (2004) Inflammation of rat dorsal root ganglia below a mid-thoracic spinal transection. Neuroreport 15:1783-1786.

Miller RJ, Jung H, Bhangoo SK, White FA (2009) Cytokine and chemokine regulation of sensory neuron function. Handb Exp Pharmacol 417-449.

Mills CD, Hains BC, Johnson KM, Hulsebosch CE (2001) Strain and model differences in behavioral outcomes after spinal cord injury in rat. J Neurotrauma 18:743-756.
Nesic O, Lee J, Johnson KM, Ye Z, Xu GY, Unabia GC, Wood TG, McAdoo DJ, Westlund KN, Hulsebosch CE, Regino Perez-Polo J (2005) Transcriptional profiling of spinal cord injury-induced central neuropathic pain. J Neurochem 95:998-1014.

Pitcher GM, Henry JL (2008) Governing role of primary afferent drive in increased excitation of spinal nociceptive neurons in a model of sciatic neuropathy. Exp Neurol 214:219-228.

Radtke C, Vogt PM, Devor M, Kocsis JD (2010) Keratinocytes acting on injured afferents induce extreme neuronal hyperexcitability and chronic pain. Pain 148:94-102.

Siddall PJ (2009) Management of neuropathic pain following spinal cord injury: now and in the future. Spinal Cord 47:352-359.

Siddall PJ, McClelland JM, Rutkowski SB, Cousins MJ (2003) A longitudinal study of the prevalence and characteristics of pain in the first 5 years following spinal cord injury. Pain 103:249-257.

Song XJ, Wang ZB, Gan Q, Walters ET (2006) cAMP and cGMP contribute to sensory neuron hyperexcitability and hyperalgesia in rats with dorsal root ganglia compression. J Neurophysiol 95:479-492.

Stucky CL, Lewin GR (1999) Isolectin $B_{4}$-positive and -negative nociceptors are functionally distinct. J Neurosci 19:6497-6505.

Study RE, Kral MG (1996) Spontaneous action potential activity in isolated dorsal root ganglion neurons from rats with a painful neuropathy. Pain 65:235-242.

Sukhotinsky I, Ben-Dor E, Raber P, Devor M (2004) Key role of the dorsal root ganglion in neuropathic tactile hypersensibility. Eur J Pain 8:135-143.

Traub RJ, Allen B, Humphrey E, Ruda MA (1990) Analysis of calcitonin gene-related peptide-like immunoreactivity in the cat dorsal spinal cord and dorsal root ganglia provide evidence for a multisegmental projection of nociceptive C-fiber primary afferents. J Comp Neurol 302:562-574.

Vierck CJ Jr, Light AR (2000) Allodynia and hyperalgesia within dermatomes caudal to a spinal cord injury in primates and rodents. Prog Brain Res 129:411-428.

Waxman SG, Hains BC (2006) Fire and phantoms after spinal cord injury: $\mathrm{Na}^{+}$channels and central pain. Trends Neurosci 29:207-215.

Woolf CJ (2007) Central sensitization: uncovering the relation between pain and plasticity. Anesthesiology 106:864-867.

Woolf CJ, Ma Q (2007) Nociceptors-noxious stimulus detectors. Neuron 55:353-364.

Wu G, Ringkamp M, Hartke TV, Murinson BB, Campbell JN, Griffin JW, Meyer RA (2001) Early onset of spontaneous activity in uninjured $\mathrm{C}$-fiber nociceptors after injury to neighboring nerve fibers. J Neurosci 21:RC140(1-5).

Xiao WH, Bennett GJ (2007) Persistent low-frequency spontaneous discharge in A-fiber and C-fiber primary afferent neurons during an inflammatory pain condition. Anesthesiology 107:813-821.

Xie W, Strong JA, Meij JT, Zhang JM, Yu L (2005) Neuropathic pain: early spontaneous afferent activity is the trigger. Pain 116:243-256.

Xie W, Strong JA, Zhang JM (2009) Early blockade of injured primary sensory afferents reduces glial cell activation in two rat neuropathic pain models. Neuroscience 160:847-857.

Xu J, Brennan TJ (2010) Guarding pain and spontaneous activity of nociceptors after skin versus skin plus deep tissue incision. Anesthesiology 112:153-164.

Yezierski RP (2009) Spinal cord injury pain: spinal and supraspinal mechanisms. J Rehabil Res Dev 46:95-107.

Zhang JM, Song XJ, LaMotte RH (1999) Enhanced excitability of sensory neurons in rats with cutaneous hyperalgesia produced by chronic compression of the dorsal root ganglion. J Neurophysiol 82:3359-3366.

Zhang JM, Li H, Brull SJ (2000) Perfusion of the mechanically compressed lumbar ganglion with lidocaine reduces mechanical hyperalgesia and allodynia in the rat. J Neurophysiol 84:798-805.

Zhao P, Waxman SG, Hains BC (2007) Extracellular signal-regulated kinase-regulated microglia-neuron signaling by prostaglandin E2 contributes to pain after spinal cord injury. J Neurosci 27:2357-2368

Zheng JH, Walters ET, Song XJ (2007) Dissociation of dorsal root ganglion neurons induces hyperexcitability that is maintained by increased responsiveness to cAMP and cGMP. J Neurophysiol 97:15-25.

Zimmermann M (1983) Ethical guidelines for investigations of experimental pain in conscious animals. Pain 16:109-110. 